JOURNAL OF THE

AMERICAN MATHEMATICAL SOCIETY

Volume 16, Number 2, Pages 259-284

S 0894-0347(02)00418-6

Article electronically published on December 3, 2002

\title{
OBSTRUCTIONS TO NONNEGATIVE CURVATURE AND RATIONAL HOMOTOPY THEORY
}

\author{
IGOR BELEGRADEK AND VITALI KAPOVITCH
}

\section{INTRODUCTION}

According to the soul theorem of J. Cheeger and D. Gromoll [CG72, a complete open manifold of nonnegative sectional curvature is diffeomorphic to the total space of the normal bundle of a compact totally geodesic submanifold, called the soul.

A natural problem is to what extent the converse to the soul theorem holds. In other words, one asks which vector bundles admit complete nonnegatively curved metrics. Various aspects of this problem have been studied in Che73, Rig78 ÖW94. Yan95, GZ00, BK01b. In this paper we only deal with bundles over closed manifolds diffeomorphic to $C \times T$, where $C$ is simply-connected and $T$ is a standard torus. By CG72 any soul has a finite cover of this form, where $\sec (C) \geq 0$.

Until recently, obstructions to the existence of metrics with sec $\geq 0$ on vector bundles were only known for flat souls [ÖW94], which corresponds to the case when $C$ is a point. In BK01b we produced a variety of examples of vector bundles which do not admit complete metrics with sec $\geq 0$. For instance, we showed that for any $C$ and $T$ with $\operatorname{dim}(T) \geq 4$ and $k \geq 2$, there are infinitely many rank $k$ vector bundles over $C \times T$ whose total spaces admit no complete metrics with sec $\geq 0$. In all these examples $\operatorname{dim}(T)>0$, in fact no obstructions are known to the existence of complete metrics with sec $\geq 0$ on vector bundles over simply-connected nonnegatively curved manifolds.

We first explain our approach to finding obstructions in case $\operatorname{dim}(T)>0$. The main geometric ingredient is the splitting theorem in Wil00, BK01b], which says that after passing to a finite cover, the normal bundle to the soul can be taken, by a base-preserving diffeomorphism, to the product $\xi_{C} \times T$ of a vector bundle $\xi_{C}$ over $C$ with the torus $T$ (see Theorem [3.1). Then one is faced with the purely topological problem of recognizing whether a given vector bundle over $C \times T$ has this property. In other words, one needs to study the orbit of $\xi_{C} \times T$ under the action of the diffeomorphism group of $C \times T$. Since vector bundles are rationally classified by the Euler and Pontrjagin classes, the problem reduces to analyzing the action of Diffeo $(C \times T)$ on the rational cohomology algebra $H^{*}(C \times T, \mathbb{Q})$ of $C \times T$. The "Taylor expansion" in $T$-coordinates of any self-diffeomorphism of $C \times T$ gives rise to a negative degree derivation of $H^{*}(C, \mathbb{Q})$. One of the main points of this paper is that the orbit of $\xi_{C} \times T$ consists of bundles of the same form, unless there exists a negative degree derivation of $H^{*}(C, \mathbb{Q})$ that does not vanish on the Euler or

Received by the editors October 28, 2001.

2000 Mathematics Subject Classification. Primary 53C20, 55P62.

Key words and phrases. Nonnegative curvature, soul, derivation, Halperin's conjecture.

(C)2002 American Mathematical Society 
Pontrjagin classes of $\xi_{C}$. In particular, if $H^{*}(C, \mathbb{Q})$ has no nonzero negative degree derivations, the above topological problem gets solved, which immediately implies that "most" bundles over $C \times T$ admit no complete metric of sec $\geq 0$.

To state our main results we need the following technical definition. Given a vector bundle $\xi$ over $C \times T$, we say that $\xi$ virtually comes from $C$ if for some finite cover $p: T \rightarrow T$, the pullback of $\xi$ by $\operatorname{id}_{C} \times p$ is isomorphic to the product $\xi_{C} \times T$ where $\xi_{C}$ is a bundle over $C$.

If $\xi$ virtually comes from $C$, then no known method can rule out the existence of a complete metric with sec $\geq 0$ on the total space $E(\xi)$ of $\xi$, and potentially all such bundles might be nonnegatively curved.

In this paper we show that the converse is often true, namely, under various assumptions on $C$, we show that, if $\xi$ is a vector bundle over $C \times T$ such that $E(\xi)$ admits a complete metric with sec $\geq 0$, then $\xi$ virtually comes from $C$. This happens for any $C$ if $\xi$ has rank two.

Theorem 1.1. Let $C$ be a closed smooth simply-connected manifold, and let $T$ be a torus. Let $\xi$ be a rank two vector bundle over $C \times T$. If $E(\xi)$ admits a complete metric with $\mathrm{sec} \geq 0$, then $\xi$ virtually comes from $C$.

Oriented $\mathbb{R}^{2}$-bundles over $C \times T$ are in one-to-one correspondence via the Euler class with $H^{2}(C \times T, \mathbb{Z}) \cong H^{2}(C, \mathbb{Z}) \oplus H^{2}(T, \mathbb{Z})$. Thus, any oriented $\mathbb{R}^{2}$-bundle $\xi$ over $C \times T$ can be written uniquely as $c_{\xi}+t_{\xi}$ where $c_{\xi} \in H^{2}(C, \mathbb{Z}), t_{\xi} \in H^{2}(T, \mathbb{Z})$. Theorem 1.1 implies that if $\sec (E(\xi)) \geq 0$, then $t_{\xi}=0$.

More generally, it is easy to see that "most" vector bundles over $C \times T$ do not virtually come from $C$, at least when $\operatorname{dim}(T)$ is large enough (for a precise result, see [BK01b, 4.4, 4.6] and Lemma B.1 below). In fact, $\xi$ virtually comes from $C$ iff all rational characteristic classes of $\xi$ lie in the $H^{*}(C, \mathbb{Q}) \otimes H^{0}(T, \mathbb{Q})$-term of the Künneth decomposition $\bigoplus_{i} H^{*}(C, \mathbb{Q}) \otimes H^{i}(T, \mathbb{Q})$ of $H^{*}(C \times T, \mathbb{Q})$.

One of the main sources of examples of closed manifolds of nonnegative curvature is given by homogeneous spaces or, more generally, biquotients of compact Lie groups. In this case we prove

Theorem 1.2. Let $C=G / / H$ be a simply-connected biquotient of compact Lie groups such that $H$ is semisimple, and let $T$ be a torus. Let $\xi$ be a vector bundle over $C \times T$ of rank $\leq 4$. If $E(\xi)$ admits a complete metric with $\sec \geq 0$, then $\xi$ virtually comes from $C$.

Let $\mathcal{H}$ be the class of simply-connected finite $\mathrm{CW}$-complexes whose rational cohomology algebras have no nonzero derivations of negative degree.

Theorem 1.3. Let $C \in \mathcal{H}$ be a closed smooth manifold, and let $T$ be a torus. If $\xi$ is a vector bundle over $C \times T$ such that $E(\xi)$ admits a complete metric with $\sec \geq 0$, then $\xi$ virtually comes from $C$.

For example, $\mathcal{H}$ contains any compact simply-connected Kähler manifold Mei83 and any compact homogeneous space $G / H$ such that $G$ is a compact connected Lie group and $H$ is a closed subgroup with $\operatorname{rank}(H)=\operatorname{rank}(G)$ ST87. It was proved in [Mar90] that the total space of a fibration belongs to $\mathcal{H}$ provided the base and the fiber do also.

Recall that a finite simply-connected cell complex $C$ is called elliptic if all but finitely many homotopy groups of $C$ are finite. If $C$ is elliptic, then $C$ has nonnegative Euler characteristic, and the sum of the Betti numbers of $C$ is $\leq 2^{m}$ where $m$ is the cohomological dimension of $C$ Fél89. 
Any compact simply-connected homogeneous space or biquotient is elliptic, and more generally, all known closed simply-connected nonnegatively curved manifolds are elliptic. In fact, it is conjectured GH83 that any closed simply-connected nonnegatively curved manifold is elliptic. If true, the conjecture would imply a classical conjecture of Chern-Hopf that nonnegatively curved manifolds have nonnegative Euler characteristic, and a conjecture of Gromov that the sum of the Betti numbers of a compact nonnegatively curved $m$-manifold is $\leq 2^{m}$.

Halperin conjectured that any elliptic space $C$ of positive Euler characteristic belongs to $\mathcal{H}$. This conjecture, which is considered one of the central problems in rational homotopy theory, has been confirmed in several important cases [FHT01. page 516]. Note that if the above conjectures are true, then $\mathcal{H}$ contains any simplyconnected compact nonnegatively curved manifold of positive Euler characteristic.

We refer to the body of the paper for other results similar to Theorems 1.1 1.3. In particular, in Section 6 we establish analogs of Theorem 1.3 for $C$ 's that belong to several classes of sphere bundles. Note that sphere bundles over closed nonnegatively curved manifolds are potentially a good source of compact manifolds with sec $\geq 0$, because the unit sphere bundle of the normal bundle to the soul is nonnegatively curved GW00. Also in Section 8, we prove an analog of Theorem 1.3 where $C$ is any currently known simply-connected positively curved manifold.

Not every nonnegatively curved vector bundle over $C \times T$ virtually comes from $C$, even though finding an explicit counterexample is surprisingly difficult. In fact, the conclusion of Theorem 1.2 fails already for rank six bundles over homogeneous spaces.

Theorem 1.4. Let $C=S U(6) /(S U(3) \times S U(3))$ and $\operatorname{dim}(T) \geq 2$. Then there exists a rank six vector bundle $\xi$ over $C \times T$ which does not virtually come from $C$, but $E(\xi)$ admits a complete metric of $\mathrm{sec} \geq 0$ such that the zero section is a soul.

To prove the above theorem, we find a nonnegatively curved vector bundle $\xi_{C}$ over $C$ with the zero section being a soul, and a negative degree derivation $D$ of $H^{*}(C, \mathbb{Q})$ that is induced by a derivation of the minimal model of $C$, and furthermore such that $D$ does not vanish on the Euler class of $\xi_{C}$, but vanishes on the Pontrjagin classes of the tangent bundle of $C$. Finding such $\xi_{C}$ and $D$ is not easy, and what makes it work here are some very special properties of the minimal model of $C$. Incidentally, $S U(6) /(S U(3) \times S U(3))$ is one of the simplest nonformal homogeneous spaces.

Now since $D$ is induced by a derivation of the minimal model, a multiple of $D$ can be "integrated" to a self-homotopy equivalence $f$ of $C \times T$. Furthermore, $f$ preserves the Pontrjagin classes of the tangent bundle of $C \times T$, because $D$ vanishes on the Pontrjagin classes of $T C$. Then by a surgery-theoretic argument, some iterated power of $f$ is homotopic to a diffeomorphism. Finally, since $D$ does not vanish on the Euler class of $\xi_{C}$, the $f$-pullback of $\xi_{C} \times T$ does not virtually come from $C$, yet it carries the pullback metric of sec $\geq 0$ with zero section being a soul.

Structure of the paper. Section 2 is a list of notations and conventions we use throughout the paper.

In Section 3 we introduce a purely topological property of a triple $(C, T, k)$, which we call splitting rigidity. As a link to nonnegative curvature, we show that if $(C, T, k)$ is splitting rigid, and $\xi$ is a rank $k$ vector bundle over $C \times T$ such that $E(\xi)$ admits a complete metric with $\sec \geq 0$, then $\xi$ virtually comes from $C$. 
In Section 4 we relate splitting rigidity to the absence of negative degree derivations of the cohomology algebra of $C$. Sections [5- 8] are devoted to applications; in particular, here we prove the results stated in Section 1 as well as splitting rigidity for certain sphere bundles and for all known positively curved manifolds.

Section 9 is an in-depth study of splitting rigidity. In particular, we prove that if $k$ is sufficiently large, then splitting rigidity can be expressed in rational homotopytheoretic terms. Section 10 contains the proof of Theorem 1.4.

In Section 11 we show by example that if $k$ is small, then changing $C$ within its homotopy type may turn a splitting rigid triple into a nonsplitting rigid one. In Section 12 we obtain stronger obstructions to nonnegative curvature on a vector bundle under the assumption that the zero section is a soul.

In Section 13 we pose and discuss several open problems. The appendix contains a surgery-theoretic lemma and an existence result for vector bundles with prescribed Euler and Pontrjagin classes.

Much of the paper can be read without any knowledge of rational homotopy theory. In fact, a rational homotopy background is only needed for Sections 7, 8, 9, 10, and 13

\section{Notation AND CONVENTIONS}

Unless stated otherwise, and all (co)homology groups have rational coefficients, all characteristic classes are over rationals, all manifolds and vector bundles are smooth.

Given a cell complex $X$, define $C h a r(X, k)$ to be the subspace of $H^{*}(X)$ equal to $\bigoplus_{i=1}^{m} H^{4 i}(X)$ if $k=2 m+1$ and equal to $\left(\bigoplus_{i=1}^{m-1} H^{4 i}(X)\right) \oplus H^{2 m}(X)$ if $k=2 m$. If $\xi$ is a (real) oriented rank $k$ vector bundle over $X$, then in case $k$ is odd, $\operatorname{Char}(X, k)$ contains the Pontrjagin classes $p_{1}(\xi), \ldots, p_{m}(\xi)$, and in case $k$ is even, $\operatorname{Char}(X, k)$ contains the Pontrjagin classes $p_{1}(\xi), \ldots, p_{m-1}(\xi)$ and the Euler class $e(\xi)$. The total Pontrjagin class $\sum_{i \geq 0} p_{i}(\xi)$ is denoted by $p(\xi)$.

For the product $X \times \bar{Y}$ of pointed spaces $X, Y$, we denote the projections of $X \times Y$ onto $X, Y$ by $\pi_{X}, \pi_{Y}$. The basepoints define the inclusions of $X, Y$ into $X \times Y$ which we denote by $i_{X}, i_{Y}$. For a map $f: X \times Y \rightarrow X^{\prime} \times Y^{\prime}$, we define $f_{X X^{\prime}}=\pi_{X^{\prime}} \circ f \circ i_{X}$ and $f_{Y Y^{\prime}}=\pi_{Y^{\prime}} \circ f \circ i_{Y}$.

For the rest of the paper, $C$ stands for a closed, connected, simply-connected, smooth manifold, and $T$ stands for a torus of some positive dimension. We use the Künneth isomorphism $H^{*}(C \times T) \cong H^{*}(C) \otimes H^{*}(T)$ to identify $\pi_{C}^{*}\left(H^{*}(C)\right)$ with the subalgebra $H^{*}(C) \otimes 1$. We denote the total space of a vector bundle $\xi$ by $E(\xi)$.

\section{SplitTing CRITERION}

The main geometric ingredient used in this paper is the following splitting theorem proved in [BK01b] (cf. Wil00]).

Theorem 3.1. Given a soul $S$ of an open complete nonnegatively curved manifold $M$, there is a finite cover $p: \tilde{M} \rightarrow M$, a soul $\tilde{S}$ of $\tilde{M}$ satisfying $p(\tilde{S})=S$, and a diffeomorphism $f: \tilde{S} \rightarrow C \times T$, where $C$ is a simply-connected manifold with $\sec (C) \geq 0$ and $T$ is a torus, such that the normal bundle to $\tilde{S}$ is the $f$-pullback of the bundle $\xi_{C} \times T$, where $\xi_{C}$ is a vector bundle over $C$ whose total space admits a metric of nonnegative curvature with the zero section being a soul. 
Let $\xi$ be a vector bundle over $C \times T$. We say that $\xi$ satisfies $(*)$ if $E(\xi)$ has a finite cover diffeomorphic to the product of $T$ and the total space of a vector bundle over a closed simply-connected manifold.

We seek to understand how assumption $(*)$ restricts $\xi$. In particular, we want to find conditions on $C$ ensuring that if $\xi$ satisfies $(*)$, then $\xi$ virtually comes from $C$.

Definition 3.2. A triple $(C, T, k)$, where $k>0$ is an integer, is called splitting rigid, if any rank $k$ vector bundle $\xi$ over $C \times T$ that satisfies $(*)$ virtually comes from $C$.

By Theorem 3.1 if $\sec (E(\xi)) \geq 0$, then $\xi$ satisfies $(*)$, so we get:

Proposition 3.3. If $(C, T, k)$ is splitting rigid and $\xi$ is a rank $k$ vector bundle over $C \times T$ such that $E(\xi)$ has a complete metric with $\mathrm{sec} \geq 0$, then $\xi$ virtually comes from $C$.

Thus if $(C, T, k)$ is splitting rigid, then the total spaces of "most" rank $k$ vector bundles over $C \times T$ do not admit complete metrics with sec $\geq 0$.

As we prove in Section 9 splitting rigidity can often be expressed in rational homotopy-theoretic terms. For example, if $k \geq \operatorname{dim}(C)$, then a triple $(C, T, k)$ is splitting rigid if and only if, for any derivation of the minimal model of $C$ that commutes with the differential and has degree within $[-\operatorname{dim}(T), 0)$, the induced derivation on $H^{*}(C)$ vanishes on $\operatorname{Char}(C, k)$.

The same statement holds with some other assumptions in place of $k \geq \operatorname{dim}(C)$, such as " $p_{i}(T C) \in C h a r(C, k)$ for all $i>0$ ". In Section 11, we give an example of when the "only if" part fails for $k<\operatorname{dim}(C)$. On the other hand, the "if" part is true without any assumptions on $k$. As a first step towards these results, we prove the following proposition, whose weak converse is obtained in Section 9.

Proposition 3.4. If any self-homotopy equivalence of $C \times T$ maps $C h a r(C, k) \otimes 1$ to itself, then $(C, T, k)$ is splitting rigid.

Proof. To check that $(C, T, k)$ is splitting rigid, we need to start with an arbitrary rank $k$ vector bundle $\xi$ over $C \times T$ that satisfies $(*)$ and prove that $\xi$ virtually comes from $C$. Without loss of generality, we can pass to a finite cover to assume that $E(\xi)$ is the total space of a vector bundle $\eta$, which is the product of $T$ and a vector bundle over a closed smooth simply-connected manifold $C^{\prime}$. In other words, $\eta$ is the $\pi_{C^{\prime}}$-pullback of a vector bundle over $C^{\prime}$.

Fix base points in $C, C^{\prime}, T$ so that the inclusions $i_{C}, i_{C}^{\prime}, i_{T}$ are defined, and let $B=C \times T, S=C^{\prime} \times T$. We think of $\xi$ and $\eta$ as two vector bundle structures on a fixed manifold $N$, we use the zero sections to identify $B$ and $S$ with smooth submanifolds of $N$, and we identify $\xi, \eta$ with the normal bundles to $B$ and $S$. Note that both $C$ and $C^{\prime}$ are homotopy equivalent to the universal cover of $N$.

Let $g: B \rightarrow S$ be the homotopy equivalence induced by the zero section of $\xi$ followed by the projection of $\eta$. Note that $\eta$ is orientable, as the pullback of a bundle over a simply-connected manifold. Fix orientations of $S$ and $\eta$, which define an orientation on $E(\eta)=E(\xi)$. We orient $B$ so that $\operatorname{deg}(g)=1$, which defines an orientation on $\xi$.

To simplify notations, assume that $k=2 m$; the case of odd $k$ is similar. Since $\xi$ has $\operatorname{rank} k, e(\xi), p_{i}(\xi) \in C h a r(B, k)$ for $0<i<m$. As we remarked in [BK01b, 4.4], the bundle $\xi$ virtually comes from $C$ iff $e(\xi)$ and $p(\xi)$ lie in $H^{*}(C) \otimes 1 \subset H^{*}(C \times T)$. 
Alternatively, since $\operatorname{Char}(B, k) \cap\left(H^{*}(C) \otimes 1\right)=\operatorname{Char}(C, k) \otimes 1$, we see that $\xi$ virtually comes from $C$ iff $e(\xi), p_{i}(\xi) \in \operatorname{Char}(C, k) \otimes 1$ for $0<i<m$.

By Whitehead's theorem, the maps $g_{C C^{\prime}}: C \rightarrow C^{\prime}, g_{T T}: T \rightarrow T$ are homotopy equivalences. Fix their homotopy inverses $g_{C C^{\prime}}^{-1}, g_{T T}^{-1}$. Consider a self-homotopy equivalence $h=\left(g_{C C^{\prime}}^{-1} \times g_{T T}^{-1}\right) \circ g$ of $C \times T$. Note that each of the maps $h_{C C}, h_{T T}$ is homotopic to the identity because, say, $h_{C C}$ is equal to

$\pi_{C} \circ h \circ i_{C} \simeq \pi_{C} \circ\left(g_{C C^{\prime}}^{-1} \times g_{T T}^{-1}\right) \circ g \circ i_{C} \simeq g_{C C^{\prime}}^{-1} \circ \pi_{C^{\prime}} \circ g \circ i_{C} \simeq g_{C C^{\prime}}^{-1} \circ g_{C C^{\prime}} \simeq \mathrm{id}_{C}$.

Now it is routine to check that $g^{*}$ maps $\operatorname{Char}\left(C^{\prime}, k\right) \otimes 1$ into $\operatorname{Char}(C, k) \otimes 1$ if and only if $h^{*}$ maps $C h a r(C, k) \otimes 1$ to itself.

By assumption $h^{*}$ maps $\operatorname{Char}(C, k) \otimes 1$ to itself, so $g^{*}\left(\operatorname{Char}\left(C^{\prime}, k\right) \otimes 1\right)=$ $\operatorname{Char}(C, k) \otimes 1$. It was observed in [BK01b, section 3] that $g^{*}$ maps $e(\eta)$ to $e(\xi)$. Thus $e(\xi) \in \operatorname{Char}(C, k) \otimes 1$, as needed, and it remains to show that $\operatorname{Char}\left(C^{\prime}, k\right) \otimes 1$ also contains $p_{i}(\xi)$ for $0<i<m$.

Since $g$, viewed as a map $B \rightarrow N$, is homotopic to the inclusion $B \hookrightarrow N$, we have that $\left.T N\right|_{B} \cong g^{\#}\left(\left.T N\right|_{S}\right)$. By the Whitney sum formula

$$
p(T B) p(\xi)=p\left(\left.T N\right|_{B}\right)=p\left(g^{\#}\left(\left.T N\right|_{S}\right)\right)=p\left(g^{\#}(T S \oplus \eta)\right)=g^{*}(p(T S)) g^{*}(p(\eta)) .
$$

Since $T$ is parallelizable, $p(T B)=p(T C) \otimes 1 \in H^{*}(C) \otimes 1$ and $p(T S)=p\left(T C^{\prime}\right) \otimes 1 \in$ $H^{*}\left(C^{\prime}\right) \otimes 1$. Since $p(T B)$ is a unit in $H^{*}(C) \otimes 1$, we can write $p(T B)^{-1}=\sum_{j} a_{j} \otimes 1$ for some $a_{j} \in H^{*}(C)$, and

$$
\begin{aligned}
p(\xi) & =p(T B)^{-1} g^{*}(p(T S)) g^{*}(p(\eta)) \\
& =\left(\sum_{j \geq 0} a_{j} \otimes 1\right)\left(\sum_{l \geq 0} g^{*}\left(p_{l}(T C) \otimes 1\right)\right)\left(\sum_{n \geq 0} g^{*}\left(p_{n}(\eta)\right)\right) .
\end{aligned}
$$

Also $p_{m}(\eta) \in H^{*}\left(C^{\prime}\right) \otimes 1$, because $\eta$ is a product of a torus and a bundle over $C^{\prime}$. By definition of "Char", this means that $p_{l}\left(T C^{\prime}\right) \otimes 1$ and $p_{n}(\eta)$ lie in $\operatorname{Char}\left(C^{\prime}, k\right) \otimes 1$ for any $0<l, n<m$, and therefore, $g^{*}\left(p_{l}\left(T C^{\prime}\right) \otimes 1\right), g^{*}\left(p_{n}(\eta)\right) \in \operatorname{Char}(C, k) \otimes 1$ for any $0<l, n<m$. The above formula now implies that $p_{i}(\xi) \in \operatorname{Char}(C, k) \otimes 1$ for $0<i<m$, as promised. This completes the proof that $\xi$ virtually comes from C.

Remark 3.5. It is easy to see that the assumption of Proposition 3.4 that $\operatorname{Char}(C, k) \otimes 1$ is invariant under any self-homotopy equivalence of $C \times T$ is equivalent to the formally weaker assumption that $\operatorname{Char}(C, k) \otimes 1$ is invariant under any self-homotopy equivalence of $C \times T$ satisfying $h_{C C} \sim \operatorname{id}_{C}, h_{T T} \sim \operatorname{id}_{T}$.

Remark 3.6. Proposition 3.4 implies that if $\operatorname{Char}(C, k) \otimes 1$ is invariant under any graded algebra automorphism of $H^{*}(C \times T)$, then $(C, T, k)$ is splitting rigid, and this is how we establish splitting rigidity in this paper, except for one example in Section [1] where deeper manifold topology gets involved.

Example 3.7. Of course, Proposition 3.4 applies if $\operatorname{Char}(C, k)=0$. Thus, $\left(S^{2 m+1}, T, k\right)$ is splitting rigid for any $k, T$.

In a special case $e(\xi) \in H^{*}(C) \otimes 1$, the very same proof of Proposition 3.4 yields the following stronger statement, in which $\epsilon^{m}$ denotes the trivial rank $m$ bundle over $C \times T$. 
Proposition 3.8. Let $\xi$ be a rank $k$ vector bundle over $C \times T$ with $e(\xi) \in H^{*}(C) \otimes 1$ such that $\xi \oplus \epsilon^{m}$ satisfies $(*)$ for some $m \geq 0$. If any self-homotopy equivalence of $C \times T$ maps $C h a r(C, k) \otimes 1$ to itself, then $\xi$ virtually comes from $C$.

The assumption on $e(\xi)$ cannot be dropped: for example, if $\xi$ is a rank two bundle over the 2-torus with $e(\xi) \neq 0$, then $\xi \oplus \epsilon^{1}$ becomes trivial in a finite cover, but $\xi$ does not. Of course, if $e(\xi)=0$ (which, for example, is always true if $k$ is odd), then $e(\xi) \in H^{*}(C) \otimes 1$.

\section{TAYLOR EXPANSION IN COHOMOLOGY}

Let $A=\bigoplus_{p} A_{p}$ be a graded $\mathbb{Q}$-algebra. If $a \in A_{p}$, we refer to $p$ as the degree of $a$ and denote it by $|a|$. In this paper we only consider graded commutative algebras with an identity element $1 \in A_{0}$, and such that $A_{p}=0$ for $p<0$.

Let $B$ be a subalgebra of $A$, and let $n \in \mathbb{Z}$. A degree $n$ derivation of $B$ with values in $A$ is a linear map $D: B \rightarrow A$ such that if $a \in A_{p}$, then $|D(a)|=p+n$, and $D(a b)=D(a) b+a D(b)(-1)^{n p}$ for any $a \in A_{p}, b \in A$. We refer to $n$ as the degree of $D$ and denote it by $|D|$. If $B=A$, we just say that $D$ is a degree $n$ derivation of $A$.

Let $\operatorname{Der}_{n}(B, A)$ be the $\mathbb{Q}$-vector space of degree $n$ derivation of $B$ with values in $A$, and write $\operatorname{Der}_{n}(A)$ for $\operatorname{Der}_{n}(A, A)$. Let $\operatorname{Der}_{-}(A)=\bigoplus_{n<0} \operatorname{Der}_{n}(A)$. We refer to derivations of $A$ of negative degree (i.e., to elements of $\operatorname{Der}_{-}(A)$ ) as negative derivations of $A$.

The cohomology algebra $H^{*}(T)$ of $T$ is an exterior algebra on degree one generators $x_{j}$ with $j=1, \ldots, \operatorname{dim}(T)$. The $\mathbb{Q}$-vector space $H^{*}(T)$ has an obvious basis $\left\{t_{i}\right\}, i=0, \ldots, n$, of square-free monomials in variables $x_{j}$ where $n=2^{\operatorname{dim}(T)}-1$. We order $\left\{t_{i}\right\}$ lexicographically so that $t_{0}=1, t_{j}=x_{j}$ for $j=1, \ldots, \operatorname{dim}(T)$, and $t_{n}=x_{1} \cdots x_{\operatorname{dim}(T)}$. Thus, $t_{i}^{2}=0$ for $i>0$. We write $H^{*}(T)=\bigoplus_{i} \mathbb{Q} t_{i}$. Then

$$
H^{*}(C \times T)=H^{*}(C) \otimes H^{*}(T)=\bigoplus_{i} H^{*}(C) \otimes \mathbb{Q} t_{i}
$$

is a free $H^{*}(C)$-module.

Let $h$ be a self-homotopy equivalence of $C \times T$. Since $H^{*}(C \times T)$ is a free $H^{*}(C)$ module with basis $\left\{t_{i}\right\}$, given $a \in H^{*}(C)$, there is a unique sequence of elements $\frac{\partial h^{*}}{\partial t_{i}}(a) \in H^{*}(C)$ such that $h^{*}(a \otimes 1)=\sum_{i}\left(1 \otimes t_{i}\right)\left(\frac{\partial h^{*}}{\partial t_{i}}(a) \otimes 1\right)$. We think of $\frac{\partial h^{*}}{\partial t_{i}}$ as $\mathbb{Q}$-linear self-maps of $H^{*}(C)$. Informally, it is useful to interpret the above formula as a Taylor expansion of $h^{*}$ at $a \otimes 1$.

Since $h^{*}$ is an algebra isomorphism, the maps $\frac{\partial h^{*}}{\partial t_{i}}$ satisfy certain recursive identities, obtained from $h^{*}(a b)=h^{*}(a) h^{*}(b)$ by collecting the terms next to $1 \otimes t_{i}$ 's. For example, $\frac{\partial h^{*}}{\partial t_{0}}$ is an algebra isomorphism of $H^{*}(C)$, and

$$
\frac{\partial h^{*}}{\partial t_{1}}(a b)=\frac{\partial h^{*}}{\partial t_{1}}(a) \frac{\partial h^{*}}{\partial t_{0}}(b)+(-1)^{-|a|} \frac{\partial h^{*}}{\partial t_{0}}(a) \frac{\partial h^{*}}{\partial t_{1}}(b) .
$$

If $h_{C C} \sim \mathrm{id}_{C}$, which can always be arranged in our case by Remark 3.5, then $\frac{\partial h^{*}}{\partial t_{0}}(a)=a$, and therefore, $\frac{\partial h^{*}}{\partial t_{1}}$ is a derivation of $H^{*}(C)$ of degree -1 .

Let $d=\operatorname{dim} T$. Suppose all degree -1 partial derivatives $\frac{\partial h^{*}}{\partial t_{1}}, \ldots, \frac{\partial h^{*}}{\partial t_{d}}$ vanish. Then we claim that $\frac{\partial h^{*}}{\partial t_{d+1}}$ is a derivation. Indeed, since $h^{*}$ is a homomorphism we 
get

$$
\begin{aligned}
a b \otimes 1 & +\frac{\partial h^{*}}{\partial t_{d+1}}(a b) \otimes t_{d+1}+\text { higher order terms } \\
= & h^{*}(a b \otimes 1)=h^{*}(a \otimes 1) h^{*}(b \otimes 1) \\
= & \left(a \otimes 1+\frac{\partial h^{*}}{\partial t_{d+1}}(a) \otimes t_{d+1}+\text { h. o. terms }\right) \\
& \times\left(b \otimes 1+\frac{\partial h^{*}}{\partial t_{d+1}}(b) \otimes t_{d+1}+\text { h. o. terms }\right) \\
= & a b \otimes 1+\left[\frac{\partial h^{*}}{\partial t_{d+1}}(a) b+a \frac{\partial h^{*}}{\partial t_{d+1}}(b)\right] \otimes t_{d+1}+\text { h. o. terms, }
\end{aligned}
$$

which proves our assertion. Similarly, if $\frac{\partial h^{*}}{\partial t_{i}}=0$ for $0<i<k$, then $\frac{\partial h^{*}}{\partial t_{k}}$ is a derivation of degree $-\left|t_{k}\right|$.

More generally, if $h_{C C}$ is not homotopic to $\operatorname{id}_{C}$, then $\frac{\partial h^{*}}{\partial t_{1}} \circ\left(\frac{\partial h^{*}}{\partial t_{0}}\right)^{-1}$ is a derivation of $H^{*}(C)$ of degree -1 , and if $\frac{\partial h^{*}}{\partial t_{i}}=0$ for $0<i<k$, then $\frac{\partial h^{*}}{\partial t_{k}} \circ\left(\frac{\partial h^{*}}{\partial t_{0}}\right)^{-1}$ is a derivation of $H^{*}(C)$ of degree $-\left|t_{k}\right|$.

Thus, if $H^{*}(C)$ has no nonzero negative derivations, then $h^{*}(a \otimes 1)=a \otimes 1$ for any $a \in H^{*}(C)$ and any self-homotopy equivalence $h$ of $C \times T$. Thus, $(C, T, k)$ is splitting rigid for any $T, k$. Combining this with Propositions 3.3 and 3.4 , we deduce Theorem 1.3 We actually need the following stronger statement.

Proposition 4.1. If every negative derivation of $H^{*}(C)$ vanishes on $C$ har $(C, k)$, then $(C, T, k)$ is splitting rigid for any $T$.

Proof. Let $h$ be a self-homotopy equivalence of $C \times T$ with $h_{C C} \sim \operatorname{id}_{C}$, so that $\frac{\partial h^{*}}{\partial t_{0}}(a)=a$, and $\frac{\partial h^{*}}{\partial t_{1}}$ is a derivation of $H^{*}(C)$ of degree -1 . By Proposition 3.4 and Remark 3.5, it suffices to show that $h^{*}(b \otimes 1)=b \otimes 1$, for all $b \in \operatorname{Char}(C, k)$.

Let $\phi_{1}$ be a self-map of $H^{*}(C \times T)$ defined by $\phi_{1}(a \otimes t)=a \otimes t-\left(1 \otimes t_{1}\right)\left(\frac{\partial h^{*}}{\partial t_{1}}(a) \otimes t\right)$ and for $t \in H^{*}(T), a \in H^{*}(C)$. The fact that $\frac{\partial h^{*}}{\partial t_{1}}$ is a derivation and $t_{1}^{2}=0$ implies that $\phi_{1}$ is a homomorphism (cf. (??) above). It is also easy to check that the map $a \otimes t \mapsto a \otimes t+\left(1 \otimes t_{1}\right)\left(\frac{\partial h^{*}}{\partial t_{1}}(a) \otimes t\right)$ is the inverse to $\phi_{1}$ and therefore $\phi_{1}$ is an automorphism of $H^{*}(C \times T)$.

Then

$$
\phi_{1} \circ h^{*}(a \otimes 1)=a \otimes 1+\sum_{i \geq 2}\left(1 \otimes t_{i}\right)\left(\phi_{1}^{i}(a) \otimes 1\right),
$$

where $\phi_{1}^{i}$ are linear self-maps of $H^{*}(C)$.

Now $\phi_{1}^{2}$ is a derivation of $H^{*}(C)$, so the formulas $\phi_{2}(a \otimes 1)=a \otimes 1-\left(1 \otimes t_{2}\right)\left(\phi_{1}^{2}(a)\right.$ $\otimes 1)$ and $\phi_{2}(1 \otimes t)=1 \otimes t$ for $t \in H^{*}(T), a \in H^{*}(C)$ define an automorphism $\phi_{2}$ of $H^{*}(C \times T)$. Then

$$
\phi_{2} \circ \phi_{1} \circ h^{*}(a \otimes 1)=(a \otimes 1)+\sum_{i \geq 3}\left(1 \otimes t_{i}\right)\left(\phi_{2}^{i}(a) \otimes 1\right),
$$

where $\phi_{2}^{i}$ are linear self-maps of $H^{*}(C)$ and $\phi_{2}^{3}$ is a derivation. Continuing in this fashion, we get automorphisms $\phi_{k}$ with $\phi_{k}(a \otimes 1)=a \otimes 1-\left(1 \otimes t_{i}\right)\left(\phi_{k-1}^{k}(a) \otimes 1\right)$ where $\phi_{k-1}^{k}$ is a derivation of $H^{*}(C)$, and such that $\phi_{n} \circ \cdots \circ \phi_{1} \circ h^{*}(a \otimes 1)=a \otimes 1$.

Thus $h^{*}(a \otimes 1)=\phi_{1}^{-1} \circ \cdots \circ \phi_{n}^{-1}(a \otimes 1)$. Also

$$
\phi_{k}^{-1}(a \otimes 1)=a \otimes 1+\left(1 \otimes t_{i}\right)\left(\phi_{k-1}^{k}(a) \otimes 1\right) .
$$


Now if $b \in C h a r(C, k)$, then by assumption $\phi_{k-1}^{k}(b)=0$ so that $\phi_{k}^{-1}(b \otimes 1)=b \otimes 1$. Thus, $h^{*}(b \otimes 1)=b \otimes 1$ as desired.

\section{SPLitTing RIGIDITY FOR RANK TWO BUNDLES}

The following well-known lemma is the key ingredient in the proof of Theorem 1.1 .

Lemma 5.1. Let $A \subset B$ be a finite-dimensional subalgebra of a commutative graded $\mathbb{Q}$-algebra $B$ satisfying $B_{0} \cong \mathbb{Q}$. Let $D$ be a derivation of $A$ of degree $-2 n<0$. Then $D$ vanishes on $A_{2 n}$.

Proof. Let $a \in A^{2 n}$. Since $B_{0} \cong \mathbb{Q}$ and $|D|=-2 n, D(a) \in B_{0}$ is a rational multiple of 1 . Choose a positive integer $m$ such that $a^{m}=0$ but $a^{m-1} \neq 0$ (which exists since $A$ is finite-dimensional). Since $|a|$ is even, we get $0=D\left(a^{m}\right)=m a^{m-1} D(a)$ so that $D(a)=0$.

Proof of Theorem [1.1. By Proposition [3.3] it is enough to show that $(C, T, 2)$ is splitting rigid. Then by Proposition 4.1 it suffices to show that any negative derivation of $H^{*}(C)$ vanishes on $H^{2}(C)$. Since $C$ is simply connected, negative derivations of degree $\neq-2$ automatically vanish on $H^{2}(C)$ for degree reasons. Since $H^{*}(C)$ is finite-dimensional, Lemma 5.1 implies that all derivations of degree -2 vanish on $H^{2}(C)$ as well.

\section{SPLitTing RIGIDITY FOR SPHERE BUNDLES}

In this section we establish splitting rigidity for various classes of sphere bundles. We need the following standard lemma.

Lemma 6.1. Let $A, B, C$ be graded commutative $\mathbb{Q}$-algebras such that $A$ is finitedimensional, $B$ is a subalgebra of $C$, and the algebras $A \otimes B$ and $C$ are isomorphic as $B$-modules. Let $\left.\operatorname{Der}(C)\right|_{B}$ be the image of the restriction map $\operatorname{Der}(C) \rightarrow \operatorname{Der}(B, C)$. Then $\left.\operatorname{Der}(C)\right|_{B}$ and $A \otimes \operatorname{Der}(B)$ are isomorphic as $\mathbb{Q}$-vector spaces.

Sketch of the proof. Let $\left\{a_{i}\right\}$ be a basis of the vector space $A$. Given a derivation $\left.D \in \operatorname{Der}(C)\right|_{B}$, define linear self-maps $D_{i}^{B}$ of $B$ by $D(1 \otimes b)=\sum_{i} a_{i} \otimes D_{i}^{B}(b)$. It is routine to check that $D_{i}^{B} \in \operatorname{Der}(B)$, and the correspondence $D \rightarrow \sum_{i} a_{i} \otimes D_{i}^{B}$ gives the promised isomorphism.

Remark 6.2. If in the above proof $D \in \operatorname{Der}_{-}(C)$, then $D_{i}^{B} \in \operatorname{Der}_{-}(B)$ for all $i$.

Theorem 6.3. Let $T$ be a torus, and let $C$ be a closed, simply-connected, smooth manifold which is the total space of a sphere bundle with zero Euler class and base $B \in \mathcal{H}$ satisfying $H^{\text {odd }}(B, \mathbb{Q})=0$. Then if $\xi$ is a vector bundle over $C \times T$ such that $E(\xi)$ admits a complete metric with $\mathrm{sec} \geq 0$, then $\xi$ virtually comes from $C$.

Proof. If $l$ is even, both the fiber and the base of the $S^{l}$-fibration $p: C \rightarrow B$ belong to $\mathcal{H}$, and hence $C \in \mathcal{H}$ by Mar90. Thus, we are done by Theorem 1.3

Suppose that $l$ is odd. Since the Euler class of $C \rightarrow B$ is trivial, the Serre spectral sequence of the fibration collapses at the $E_{2}$-term, so $H^{*}(C)$ and $H^{*}(B) \otimes$ $H^{*}\left(S^{l}\right)$ are isomorphic, as $H^{*}(B)$-modules. Since $H^{\text {odd }}(B)=0$, this implies that $p: H^{\text {even }}(B) \rightarrow H^{\text {even }}(C)$ is an isomorphism. Note that under the above identification of $H^{*}(C)$ and $H^{*}(B) \otimes H^{*}\left(S^{l}\right), p^{*}\left(H^{*}(B)\right)=H^{e v e n}(C)$ corresponds to $H^{*}(B) \otimes 1$. 
By Proposition 4.1 to prove splitting rigidity of $(C, T, k)$ for any $T, k$, it is enough to show that all negative derivations of $H^{*}(C)$ vanish on $H^{\text {even }}(C)$. Let $D \in \operatorname{Der}_{-}\left(H^{*}(C)\right)$ be a negative derivation. By Lemma 6.1 we can write $\left.D\right|_{H^{\text {even }}(C)}$ as $a D_{1}+D_{2}$ where $a \in H^{l}\left(S^{l}\right)$ and $D_{1}, D_{2} \in \operatorname{Der}_{-}\left(H^{*}(B)\right)$. Now $B \in \mathcal{H}$ implies that $D_{1}=D_{2}=0$, and hence $\left.D\right|_{H^{e v e n}(C)}=0$, as needed.

In case $\operatorname{dim}\left(S^{l}\right) \geq \operatorname{dim}(B)$, the Euler class is automatically zero, therefore we have

Corollary 6.4. Let $S^{l} \rightarrow C \rightarrow B$ be a sphere bundle over $B \in \mathcal{H}$ with $H^{\text {odd }}(B)=0$ where $l \geq \operatorname{dim}(B)$. Then $(C, T, k)$ is splitting rigid for any $T, k$.

Remark 6.5. If $B$ is elliptic, then $H^{\text {odd }}(B, \mathbb{Q})=0$ is equivalent to $\chi(B)>0$ [FHT01. Proposition 32.10], where $\chi$ is the Euler characteristic. In particular, all homogeneous spaces $G / H$ with $\operatorname{rank}(H)=\operatorname{rank}(G)$ have $H^{\text {odd }}(G / H, \mathbb{Q})=0$. As we mentioned in the introduction, conjecturally, any nonnegatively curved manifold $B$ of positive Euler characteristic has $H^{\text {odd }}(B, \mathbb{Q})=0$ and lies in $\mathcal{H}$.

Let $\mathcal{H}(n)$ be the class of simply-connected finite CW-complexes whose rational cohomology algebras are generated in dimension $n$. By Lemma $5.1 \mathcal{H}(2 n) \subset \mathcal{H}$ for any $n$. Notice that this implies that any $B \in \mathcal{H}(2 n)$ satisfies the assumptions of Theorem 6.3 This also implies that the class $\mathcal{H}(2 n)$ is closed under fibrations because if $F \rightarrow E \rightarrow B$ is a fibration with the fiber in $\mathcal{H}$, then by [Mar90] there is an $H^{*}(B)$-module isomorphism $H^{*}(E) \cong H^{*}(F) \otimes H^{*}(B)$. A simple MayerVietoris arguments implies that if closed simply-connected manifolds $C, C^{\prime}$ belong to $\mathcal{H}(2 n)$, then so does the connected sum $C \# C^{\prime}$.

Example 6.6. Besides being closed under fibrations and connected sums, the class $\mathcal{H}(2 n)$ contains the following nonnegatively curved manifolds:

- $\mathcal{H}(2)$ contains $S^{2}, C P^{n}, C P^{n} \# C P^{n}, C P^{n} \# \overline{C P^{n}}$ [Che73, all nontrivial $S^{2}$ bundles over $S^{4}$ GZ00, biquotients $G / / T$ of a compact connected Lie group $G$ by a maximal torus $T$ [Sin93], $S O(2 n+1) /(S O(2 n-1) \times S O(2))$ which is a homology $C P^{2 n-1}$ [MZ87, projectivized tangent bundle to $C P^{n}$ [Wil02, and the exceptional space $G_{2} / U(2)$ which is a homology $C P^{5}$ [MZ87];

- $\mathcal{H}(4)$ contains $S^{4}, H P^{n}, H P^{n} \# H P^{n}, H P^{n} \# \overline{H P^{n}}$ Che73, $G_{2} / S O(4)$ which is a homology $H P^{2}$, projectivized tangent bundle to $H P^{n}$ [Wil02], and $S p(n) / K$ where $K$ is the product of $n$ copies of $S p(1)$;

- $\mathcal{H}(8)$ contains $S^{8}, F_{4} / \operatorname{Spin}(9)$, and $F_{4} / \operatorname{Spin}(8)$.

Theorem 6.7. If $S^{l} \rightarrow C \rightarrow B$ is a sphere bundle such that $B \in \mathcal{H}(2 n)$ for some $n$, then $(C, T, k)$ is splitting rigid for any $T, k$.

Proof. If $l$ is even, then $S^{l}, B \in \mathcal{H}$ so that $C \in \mathcal{H}$ by Mar90, and we are done by Theorem 1.3. If the Euler class of $C \rightarrow B$ vanishes, then the result follows by Theorem 6.3. Thus, we can assume that $l$ is odd and $p: C \rightarrow B$ has nonzero Euler class.

It follows from the Gysin sequence that $p^{*}: H^{e v e n}(B) \rightarrow H^{e v e n}(C)$ is onto, so $H^{\text {even }}(C)$ is generated in dimension $2 n$. Again, we see from the Gysin sequence that $H^{i}(C)=0$ for $0<i<2 n$. Hence, Lemma 5.1 implies that $\operatorname{Der}_{-}\left(H^{\text {even }}(C), H^{*}(C)\right)$ $=0$. So by Proposition 4.1, $(C, T, k)$ is splitting rigid for any $T, k$. 


\section{SPlitTing RIGIDITY FOR BIQUOTIENTS}

Let $G$ be a compact Lie group and $H \leq G \times G$ be a compact subgroup. Then $H$ acts on $G$ on the left by the formula $\left(h_{1}, h_{2}\right) g=h_{1} g h_{2}^{-1}$. The orbit space of this action is called a biquotient of $G$ by $H$ and denoted by $G / / H$. If the action of $H$ on $G$ is free, then $G / / H$ is a manifold. This is the only case we consider in this paper. In the special case when $H$ has the form $K_{1} \times K_{2}$ where $K_{1} \subset G \times 1 \subset G \times G$ and $K_{2} \subset 1 \times G \subset G \times G$ we will sometimes write $K_{1} \backslash G / K_{2}$ instead of $G / /\left(K_{1} \times K_{2}\right)$. For any biinvariant metric on $G$ the above action of $H$ is isometric, and therefore, $G / / H$ can be equipped with a submersion metric, which by O'Neill's submersion formula is nonnegatively curved. Thus, biquotients form a large class of examples of nonnegatively curved manifolds.

As was observed by Eschenburg [Esc92a, any biquotient $G / / H$ is diffeomorphic to a biquotient of $G \times G$ by $G \times H$ written as $\Delta G \backslash G \times G / H$, where $\Delta G$ stands for the diagonal embedding of $G$ into $G \times G$. Let $p: G / / H \rightarrow B_{H}$ be the classifying map of the principle $H$ bundle $H \rightarrow G \rightarrow G / / H$. Then it is easy to see (cf. Esc92a]) that $G \rightarrow G / / H \rightarrow B H$ is a Serre fibration (which need not be principal!). Moreover, this fibration fits into the following fibered square (see [Esc92a] and Sin93])

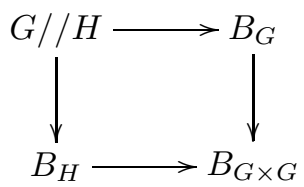

where both vertical arrows are fibrations with fiber $G$ and both horizontal arrows are fibrations with fiber $(G \times G) / H$. In particular, the fibration $G / / H \rightarrow B_{H}$ is the pullback of the fibration $G \rightarrow B_{G} \rightarrow B_{G \times G}$. Following Eschenburg, we call the fibration $B_{G} \rightarrow B_{G \times G}$ the reference fibration.

Next we are going to construct a Sullivan model of the biquotient $G / / H$.

We refer to [T097, Chapter1] for a gentle introduction to rational homotopy theory, and we use the textbook FHT01 as a comprehensive reference.

Recall that a free DGA $(\Lambda V, d)$ is called pure if $V$ is finite-dimensional and $\left.d\right|_{V^{\text {even }}}=0$ and $d\left(V^{\text {odd }}\right) \subset V^{\text {even }}$. It is well known that homogeneous spaces admit natural pure Sullivan models given by their Cartan algebras. The next proposition shows that the same remains true for biquotients.

Proposition 7.1. Let $G / / H$ be a biquotient. Then it admits a pure Sullivan model.

Proof. We begin by constructing the canonical model of the reference fibration $\phi: B_{G} \rightarrow B_{G \times G}=B_{G} \times B_{G}$. Since this fibration is induced by the diagonal map $\Delta: G \rightarrow G \times G$, it follows that $\phi$ is the diagonal embedding $\Delta_{B_{G}}: B_{G} \rightarrow B_{G} \times B_{G}$. Consider the map $\phi^{*}: H^{*}\left(B_{G} \times B_{G}\right) \rightarrow H^{*}\left(B_{G}\right)$. It is well known that $G$ is rationally homotopy equivalent to $S^{2 m_{1}-1} \times \cdots \times S^{2 m_{n}-1}$ and the minimal model of $B_{G}$ is isomorphic to $H^{*}(B G, \mathbb{Q}) \cong \mathbb{Q}\left[x_{1}, \ldots, x_{n}\right]$ with zero differentials and with $\left|x_{i}\right|=2 m_{i}$. Similarly the minimal model of $B_{G} \times B_{G}$ is isomorphic to its cohomology $\operatorname{ring} B=\mathbb{Q}\left[x_{1}, \ldots, x_{n}, y_{1}, \ldots, y_{n}\right]$ with $\left|x_{i}\right|=\left|y_{i}\right|=2 m_{i}$. Thus $\phi^{*}$ can be viewed as a DGA-homomorphism of minimal models of $B_{G}$ and $B_{G \times G}$.

Let us construct a Sullivan model of $\phi^{*}$. Since $\phi=\Delta_{B_{G}}$, we compute that $\phi^{*}\left(x_{i}\right)=\phi^{*}\left(y_{i}\right)=x_{i}$ for all $i=1, \ldots, n$. Consider the relative Sullivan algebra $\left(B \otimes \Lambda\left(q_{1}, \ldots, q_{n}\right), d\right)$ where $d x_{i}=d y_{i}=0$ and $d q_{i}=x_{i}-y_{i}$. Then it is immediate to check that this relative algebra is a Sullivan model (in fact, a minimal one) of 
$\phi^{*}$ with the quasi-isomorphism $B \otimes \Lambda\left(q_{1}, \ldots, q_{n}\right) \rightarrow H^{*}\left(B_{G}\right)$ given by $x_{i} \rightarrow x_{i}$, $y_{i} \rightarrow x_{i}, q_{i} \rightarrow 0$.

By the naturality of models of maps [FHT01, page 204, Proposition 15.8], from the fibered square (7.3), we obtain that a Sullivan model of the map $G / / H \rightarrow$ $B_{H}$ can be given by the pushout of $\left(B \otimes \Lambda\left(q_{1}, \ldots, q_{n}\right), d\right)$ via the homomorphism $f^{*}: B \rightarrow H^{*}\left(B_{H}\right)$; i.e., it can be written as

$$
\left(H^{*}\left(B_{H}\right), 0\right) \otimes_{(B, d)}\left(B \otimes \Lambda\left(q_{1}, \ldots, q_{n}\right), d\right)=\left(H^{*}\left(B_{H}\right) \otimes \Lambda\left(q_{1}, \ldots, q_{n}\right), \bar{d}\right)
$$

where $\bar{d}$ is given by $\left.\bar{d}\right|_{H^{*}\left(B_{H}\right)}=0$ and $\bar{d}\left(q_{i}\right)=f^{*}\left(x_{i}-y_{i}\right)$. In particular, $M(G / / H)=$ $\left(H^{*}\left(B_{H}\right) \otimes \Lambda\left(q_{1}, \ldots, q_{n}\right), \bar{d}\right)$ is a model for $G / / H$. Notice that $H^{*}\left(B_{H}\right)$ is a free polynomial algebra on a finite number of even-dimensional generators, and thus the model $M(G / / H)$ is pure.

Remark 7.2. It is easy to see that the minimal model of a pure Sullivan model is again pure. Therefore, Proposition 7.1 implies that minimal models of biquotients are pure.

Remark 7.3. The pure model $M(G / / H)$ constructed in the proof of Proposition 7.1 provides an effective way of computing rational cohomology of biquotients. We refer to $M(G / / H)$ as the Cartan model of $G / / H$. This method of computing $H^{*}(G / / H)$ is essentially equivalent to the method developed by Eschenburg [Esc92b] who computed the Serre spectral sequence of the fibration $G \rightarrow G / / H \rightarrow B_{H}$. In fact, it is easy to recover this spectral sequence by introducing the standard bigrading on the Cartan model $M(G / / H)$. Also note that in the case when $G / / H$ is an ordinary homogeneous space (i.e., when $H \subset G \times G$ has the form $H \times 1 \subset G \times G$ ) this model is easily seen to reduce to the standard Cartan model of $G / H$.

Proof of Theorem 1.2. By Theorem 1.1, we only have to consider the case when $k=3$ or 4 . By Proposition 3.3 it is enough to show that $(C, T, k)$ is splitting rigid for any $T$ and $k=3$ or 4 . Since $\operatorname{Char}(C, 3)=\operatorname{Char}(C, 4)=\left\langle H^{4}(C)\right\rangle$ and according to Proposition 4.1, to insure splitting rigidity, it is enough to show that all negative derivations of $H^{*}(G / / H)$ vanish on $H^{4}(G / / H)$.

By passing to a finite cover we can assume that both $G$ and $H$ are connected. Since $H$ is semisimple and $G / / H$ is simply connected, the long exact sequence of the fibration $H \rightarrow G \rightarrow G / / H$ implies that $G$ is also semisimple.

Let $(\Lambda(V), d)$ be the minimal model of $G / / H$. Since $G / / H$ is 2-connected, we have that $V^{1}=V^{2}=0$. According to Proposition 7.1 the model $(\Lambda(V), d)$ is pure. By minimality of $(\Lambda(V), d)$, we have that $\left.d\right|_{V_{3}}=0$ and $V^{3} \cong H^{3}(G / / H)$. By the structure theorem for pure DGAs, Oni94, page 141, Proposition 3], this implies that $\Lambda(V) \cong \Lambda V^{3} \otimes \Lambda(\hat{V})$ and $H^{*}(\Lambda(V)) \cong \Lambda V^{3} \otimes H^{*}(\Lambda(\hat{V}))$ for some differential subalgebra $\Lambda(\hat{V}) \subseteq \Lambda(V)$ such that $\hat{V}^{3}=0$. Let $A=\Lambda V^{3}$ and $B=H^{*}(\Lambda(\hat{V}))$. By the above, $B^{1}=B^{2}=B^{3}=0$. Therefore, by Lemma 5.1, all negative derivations of $B$ vanish on $B^{4}$. Notice that $H^{4}(G / / H)$ corresponds to $1 \otimes B^{4}$ under the isomorphism $H^{*}(G / / H) \cong A \otimes B$, and hence applying Lemma 6.1, we conclude that negative derivations of $H^{*}(G / / H)$ vanish on $H^{4}(G / / H)$.

Remark 7.4. Using Proposition [3.8, we get a stronger version of Theorem 1.2 Namely, if $\xi$ is a vector bundle over $C \times T$ of rank 3 or 4 with $e(\xi) \in H^{*}(C) \otimes 1$ such that for some $m \geq 0$, the manifold $E(\xi) \times \mathbb{R}^{m}$ admits a complete metric with sec $\geq 0$, then $\xi$ virtually comes from $C$. If $\xi$ has rank 3 , then $e(\xi)=0$, so the assumption $e(\xi) \in H^{*}(C) \otimes 1$ is automatically true. 
Remark 7.5. It would be interesting to see whether Theorem 1.2 remains true if $H$ is not assumed to be semisimple. In that case a slight modification of the proof of Theorem 1.2 still shows that derivations of degree $-4,-3$ and -1 vanish on $H^{4}(G / / H)$, and therefore $\left(G / / H, S^{1}, k\right)$ is splitting rigid for $k \leq 4$. However, as of this writing, we are unable to see whether degree -2 derivations have to vanish, and thus the general case remains unclear.

Proposition 7.6. Let $C=G / / H$ be a simply-connected biquotient of a compact group $G$ by a torus $H$ satisfying $\operatorname{rank}(H)=\operatorname{rank}(G)-1$. Then $(C, T, k)$ is splitting rigid for any $T, k$.

Proof. First, we show that $H^{\text {even }}(G / / H)$ is generated in dimension 2. Consider the Cartan model $M(G / / H)=\left(H^{*}\left(B_{H}\right) \otimes \Lambda\left(q_{1}, \ldots, q_{n}\right), \bar{d}\right)$. It admits a natural grading by the wordlength in $q_{i}$ 's given by $M(G / / H)_{k}=H^{*}\left(B_{H}\right) \otimes \Lambda^{k}\left(q_{1}, \ldots, q_{n}\right)$. Since the differential decreases the wordlength in $q_{i}$ 's by 1 , this grading induces a natural grading in the cohomology $H^{*}(G / / H)=\bigoplus H_{k}^{*}(G / / H)$; this is the so-called lower grading on $H^{*}(G / / H)$.

According to [Hal77, Theorem 2] (also cf. [Sin93] Proposition 6.4]), $H_{k}^{*}(G / / H)=$ 0 for $k>\operatorname{rank}(G)-\operatorname{rank}(H)$. In our case $\operatorname{rank}(G)-\operatorname{rank}(H)=1$, and hence $H_{k}^{*}(G / / H)=0$ for $k>1$. Next observe that $H_{1}^{\text {even }}(G / / H)=0$ since $\left|q_{i}\right|$ is odd for any $i$ and $H^{\text {odd }}\left(B_{H}\right)=0$. Similarly $H_{0}^{\text {odd }}(G / / H)=0$. Therefore, $H^{\text {even }}(G / / H)=$ $H_{0}^{*}(G / / H)$ which is equal to the quotient of $H^{*}\left(B_{H}\right)$ by $\bar{d}\left(\Lambda\left(q_{1}, \ldots, q_{n}\right)\right)$. Since $H$ is a torus, $H^{*}\left(B_{H}\right)$ is generated by 2 -dimensional classes and hence by the above the same is true for $H^{\text {even }}(G / / H)$.

By Lemma 5.1 this implies that Der_ $\left(H^{\text {even }}(G / / H), H^{*}(G / / H)\right)=0$ which by the splitting criterion Proposition 4.1 means that $(G / / H, T, k)$ is splitting rigid for any $T, k$.

\section{SPLITTING RIGIDITY FOR KNOWN POSITIVELY CURVED MANIFOLDS}

Proposition 8.1. Let $C$ be a known closed simply-connected positively curved manifold, and let $T$ be a torus. If $\xi$ is a vector bundle over $C \times T$ such that $E(\xi)$ admits a complete metric with $\mathrm{sec} \geq 0$, then $\xi$ virtually comes from $C$.

Proof. First of all, note that all known even-dimensional positively curved manifolds belong to $\mathcal{H}$. Indeed, it follows from the classification theorem of positively curved homogeneous spaces Wal72 that any even-dimensional homogeneous space belongs to $\mathcal{H}(2 n) \subset \mathcal{H}$ for some $n>0$. The only known example of a positively curved evendimensional manifold which is nondiffeomorphic to a positively curved homogeneous space is the space $M^{6}=S U(3) / / T^{2}$ [Esc92b]. This space is an $S^{2}$-bundle over $C P^{2}$ and, therefore, it lies in $\mathcal{H}$.

Now we establish splitting rigidity for all known odd-dimensional positively curved manifolds. Those are the standard spheres, the Berger 7-dimensional homology sphere $B^{7}=S p(2) / S p(1)_{\max }$, the Eschenburg 7-manifolds $E_{k, l, m, n}^{7}$ Esc82] all obtained as biquotients of $S U(3)$ by $S^{1}$, and the Bazaikin 13-manifolds $B_{k, l, m, n}^{13}$ Baz96. obtained as biquotients of $S U(5)$ by $S p(2) \times S^{1}$.

By Example 3.7 any odd-dimensional rational homology sphere is splitting rigid, so it remains to deal with the Eschenburg and Bazaikin manifolds. By Lemma 8.2 below, all Eschenburg manifolds are rationally homotopy equivalent to $S^{2} \times S^{5}$, and all Bazaikin manifolds are rationally homotopy equivalent to $C P^{2} \times S^{9}$. Since both $C P^{2}$ and $S^{2}$ belong to $\mathcal{H}(2)$, the proof of Theorem 6.3 implies that any negative 
derivation of $H^{*}\left(S^{2} \times S^{5}\right)$ or $H^{*}\left(C P^{2} \times S^{9}\right)$ vanishes on even cohomology. Now Proposition 4.1 implies splitting rigidity of all Bazaikin and Eschenburg manifolds.

Lemma 8.2. All Eschenburg manifolds are rationally homotopy equivalent to $S^{2} \times$ $S^{5}$; all Bazaikin manifolds are rationally homotopy equivalent to $C P^{2} \times S^{9}$.

Proof. We only give a proof for the Bazaikin manifolds; the Eschenburg manifolds are treated similarly. Let $B^{13}$ be a Bazaikin manifold. From the homotopy sequence of the fibration

$$
S p(2) \times S^{1} \rightarrow S U(5) \rightarrow B^{13}
$$

one easily sees that $B^{13}$ has the same rational homotopy groups as $C P^{2} \times S^{9}$. Let $M=(\Lambda V, d)$ be the minimal model of $B^{13}$. It is well known (e.g., see [FHT01] Theorem 15.11]) that $V \cong \operatorname{Hom}_{\mathbb{Z}}\left(\pi_{*}(B), \mathbb{Q}\right)$. Therefore, $M$ is a free graded algebra on generators $x_{2}, y_{5}, y_{9}$ with the degrees of the generators given by the subscripts. Obviously, $d\left(x_{2}\right)=0$ and $d\left(y_{5}\right)=k x_{2}^{3}$ for some rational $k$. Note that $k \neq 0$, else we would have $H^{5}(M) \cong \mathbb{Q}$ which is known not to be the case by Baz96. Replacing $y_{5}$ with $y_{5} / k$, we can assume that $k=1$. It is clear that $d y_{9}$ must be equal to $l x_{2}^{5}$ for some rational $l$. We claim that any such minimal model is isomorphic to the one with $l=0$. Indeed, let $M_{l}=\left\langle\Lambda\left(x_{2}, y_{5}, y_{9}\right) \mid d x_{2}=0, d y_{5}=x_{2}^{3}, d y_{9}=l x_{2}^{5}\right\rangle$. Consider the map $M_{0} \rightarrow M_{l}$ given by $x_{2} \rightarrow x_{2}, y_{5} \rightarrow y_{5}, y_{9} \rightarrow y_{9}-l y_{5} x_{2}^{2}$. This map is easily seen to be a DGA-isomorphism with the inverse given by $x_{2} \rightarrow x_{2}, y_{5} \rightarrow y_{5}, y_{9} \rightarrow$ $y_{9}+l y_{5} x_{2}^{2}$. Since $M_{0}$ is a minimal model of $C P^{2} \times S^{9}$, the proof is complete.

Remark 8.3. As we explained above, any known closed (simply-connected) evendimensional positively curved manifold $C$ belongs to $\mathcal{H}(2 n)$ for some $n$. The same is true for all known Wil02 even-dimensional manifolds with sectional curvature positive on an open dense subset, such as projectivized tangent bundles of $H P^{n}$ and $C P^{n}$. Therefore, all these examples are also splitting rigid for any $T, k$.

\section{SPLITTING RIGIDITY AND DERIVATIONS IN MINIMAL MODELS}

In this section, we study splitting rigidity using methods of rational homotopy theory. We prove that a triple $(C, T, k)$ is splitting rigid if, for any derivation of the minimal model of $C$ that commutes with differential and has degree within [- $\operatorname{dim}(T), 0)$, the induced derivation on $H^{*}(C)$ vanishes on $C h a r(C, k)$. The converse to this statement is proved in Proposition 9.4 under various assumptions on $(C, T, k)$, such as $2 k \geq \operatorname{dim}(C \times T)+3$, or $p_{i}(T C) \in \operatorname{Char}(C, k)$.

Thus, under either of the assumptions, the splitting rigidity is a phenomenon of rational homotopy theory, in other words, whether or not $(C, T, k)$ is splitting rigid depends only on $k, \operatorname{dim}(T)$, and the minimal model (or equivalently, the rational homotopy type) of $C$. This is no longer true for smaller $k$; in fact in Section[11, we give an example of two triples $(C, T, 6),(M, T, 6)$ with homotopy equivalent $C$ and $M$, such that $(C, T, 6)$ is splitting rigid, while $(M, T, 6)$ is not.

Let $\left(M_{C}, d_{C}\right),\left(M_{T}, d_{T}\right)$ be (Sullivan) minimal models for $C, T$. Since $H^{*}(T)$ is a free exterior algebra, we can assume that $M_{T}=H^{*}(T)$ and $d_{T}=0$. Then $\left(M_{C} \otimes M_{T}, d\right)$ is a minimal model for $C \times T$, where for $x \in M_{C}, t \in M_{T}$

$$
d(x \otimes t)=d_{C}(x) \otimes t+(-1)^{|x|} x \otimes d_{T}(t)=d_{C}(x) \otimes t .
$$

First, we modify the arguments of Section 4 to produce the Taylor expansion in $M_{C} \otimes M_{T}$ of any self-homotopy equivalence of $C \times T$. Let $h$ be a self-homotopy 
equivalence of $C \times T$. Then the induced map of minimal models $h^{\#}$ is an isomorphism [FHT01, 12.10(i)].

For $x \in M_{C}$, consider $\frac{\partial h^{\#}}{\partial t_{i}}(x) \in M_{C}$ such that $h^{\#}(x \otimes 1)=\sum_{i}\left(1 \otimes t_{i}\right)\left(\frac{\partial h^{\#}}{\partial t_{i}}(x) \otimes 1\right)$. Since $h^{\#}$ commutes with $d$ and $d\left(1 \otimes t_{i}\right)=0$, we get that each $\frac{\partial h^{\#}}{\partial t_{i}}$ commutes with $d_{C}$, up to sign, and therefore induces a linear self-map of $H^{*}(C)$.

As in Section 4, since $h^{\#}$ is an algebra isomorphism, the maps $\frac{\partial h^{\#}}{\partial t_{i}}$ satisfy the same recursive identities, obtained from $h^{\#}(x y)=h^{\#}(x) h^{\#}(y)$ by collecting the terms next to $1 \otimes t_{i}$ 's. Thus, $\frac{\partial h}{\partial t_{0}}$ is an isomorphism of $\left(M_{C}, d_{C}\right)$, and $\frac{\partial h^{\#}}{\partial t_{0}} \circ\left(\frac{\partial h^{\#}}{\partial t_{0}}\right)^{-1}$ is a derivation of $M_{C}$ of degree -1 . More generally, if $\frac{\partial h^{\#}}{\partial t_{i}}(x)=0$ for all $x \in M_{C}$ and all $0<i<k$, then $\frac{\partial h^{\#}}{\partial t_{k}} \circ\left(\frac{\partial h^{\#}}{\partial t_{0}}\right)^{-1}$ is a derivation of $M_{C}$ of degree $-\left|t_{k}\right|$. As we noted before, $\frac{\partial h^{\#}}{\partial t_{h}} \circ\left(\frac{\partial h^{\#}}{\partial t_{0}}\right)^{-1}$ commutes with $d_{C}$, up to sign. Hence, it induces a degree $-\left|t_{k}\right|$ derivation of $H^{*}(C)$. A slight variation of the proof of Proposition 4.1 implies the following.

Proposition 9.1. If each negative derivation of $H^{*}(C)$ induced by a derivation of $M_{C}$ of degree $\geq-\operatorname{dim}(T)$ vanishes on $C h a r(C, k)$, then $(C, T, k)$ is splitting rigid.

Remark 9.2. The assumptions of Proposition 9.1 only involve the minimal model of $C$. Thus, if $C^{\prime}$ is rationally homotopy equivalent to $C$ and $(C, T, k)$ satisfies the assumptions of Proposition 9.1, then so does $\left(C^{\prime}, T, k\right)$.

The following lemma shows that an integer multiple of any negative derivation of $M_{C}$ can be "integrated" to a self-homotopy equivalence of $C \times T$. This gives many examples of triples which are not splitting rigid and is a crucial ingredient in the proof of Theorem 1.4

Lemma 9.3. Let $D$ be a negative derivation of $H^{*}(C)$ induced by a derivation of $M_{C}$ that commutes with $d_{C}$. Let $T$ be a torus with $|D| \geq-\operatorname{dim}(T)$. Then there are integers $i, m>0$ and a self-homotopy equivalence $h$ of $C \times T$ such that $h^{*}(a \otimes 1)=a \otimes 1+\left(1 \otimes t_{i}\right)(m D(a) \otimes 1)$ for $a \in H^{*}(C)$, and $h_{C C} \sim \operatorname{id}_{C}, \pi_{T} \circ h=\pi_{T}$.

Proof. Let $\tilde{D}$ be a derivation of $M_{C}$ that induces $D$. The fact that $\tilde{D}$ is a derivation implies that the map $\phi: M_{C} \rightarrow M_{C} \otimes M_{T}$ defined by $\phi(x)=(x \otimes 1)+\left(1 \otimes t_{i}\right)(\tilde{D}(x)$ $\otimes 1)$ is a DGA-homomorphism.

Being a DGA-homomorphism, $\phi$ defines a (unique up to homotopy) map of rationalizations $f: C_{0} \times T_{0} \rightarrow C_{0}$ where we can choose $f$ so that $f \circ i_{C_{0}}=\operatorname{id}_{C_{0}}$. Look at the diagram

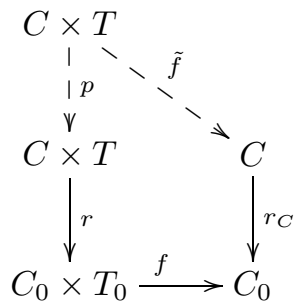

where $r=r_{T} \times r_{C}: C \times T \rightarrow C_{0} \times T_{0}$ is the rationalization, and try to find a finite covering $p$ and a map $\tilde{f}$ that makes the diagram commute and satisfies $\tilde{f} \circ i_{C}=\operatorname{id}_{C}$.

It follows from an obstruction theory argument as in [BK01b, Section 4] that such $p, \tilde{f}$ can be constructed, where the key point is that all obstructions are torsion since 
the homotopy fiber of $r$ has torsion homotopy groups, and all torsion obstruction vanish after precomposing with a suitable finite cover $p$. Furthermore (cf. the proof of Lemma B.1), one can choose $p, \tilde{f}$ satisfying $\tilde{f} \circ i_{C}=\operatorname{id}_{C}, p=\operatorname{id}_{C} \times\left(\times^{n}\right)$, where $\times^{n}: T \rightarrow T$ is the $n$th power map, so that, for some positive integer $m$, the induced map on $H^{*}(C)$ satisfies

$$
\tilde{f}^{*}[a \otimes 1]=[a \otimes 1]+m\left(1 \otimes t_{i}\right)([\tilde{D}(a)] \otimes 1) .
$$

Finally, by Whitehead's theorem, the map $h(c, t)=(\tilde{f}(c, t), t)$ is a homotopy equivalence with the desired properties.

Proposition 9.4. Assume $(C, T, k)$ is splitting rigid and either $2 k \geq \operatorname{dim}(C \times T)+3$ or $p_{i}(T C) \in C h a r(C, k)$ for all $i>0$. If $D$ is a negative derivation of $H^{*}(C)$ of degree $\geq-\operatorname{dim}(T)$ induced by a derivation of $M_{C}$, then $D$ vanishes on Char $(C, k)$.

Proof. Arguing by contradiction, let $D$ be a negative derivation of $H^{*}(C)$ with $|D| \geq-\operatorname{dim}(T)$ such that $D$ does not vanish on $C h a r(C, k)$.

First, we show that $D$ is nonzero on either Euler or Pontrjagin class of a rank $k$ bundle $\xi_{C}$ over $C$. Assume, for example, that $k=2 m$ so that $\operatorname{Char}(C, k)=$ $\left(\bigoplus_{i=1}^{m-1} H^{4 i}(C)\right) \oplus H^{2 m}(C)$. If $a \in H^{2 m}(C)$ satisfies $D(a) \neq 0$, then by Lemma B.1. $a$ is proportional to the Euler class of some rank $k$ vector bundle $\xi_{C}$ over $C$. If $a \in H^{4 i}(C)$, then by Lemma B.1 $a$ is proportional to the $i$ th Pontrjagin class of some bundle of rank $k$ vector bundle $\xi_{C}$ over $C$.

For example, suppose that $D$ is nonzero on the Euler class $e\left(\xi_{C}\right)$. By Lemma 9.3 there is a self-homotopy equivalence $f$ of $C \times T$ such that $f^{*}\left(e\left(\xi_{C}\right) \otimes 1\right)$ does not lie in $H^{*}(C) \otimes 1$. Now there are two cases to consider.

If $2 k \geq \operatorname{dim}(C \times T)+3$, then by Haefliger's embedding theorem [Hae61] the homotopy equivalence $f: C \times T \rightarrow E\left(\xi_{C}\right) \times T$ is homotopic to a smooth embed$\operatorname{ding} q$. The normal bundle $\nu_{q}$ has rank $k$. Since $q$ and $f$ are homotopic, $\nu_{q}$ and $q^{\#}\left(\xi_{C} \times T\right)$ have equal Euler and Pontrjagin classes. This follows from the intersection pairing interpretation of the Euler class and the Whitney sum formula for the total Pontrjagin class (see BK01b, Section 3] for details). Thus, $e\left(\nu_{q}\right)=$ $f^{*}\left(e\left(\xi_{C}\right) \otimes 1\right)$ does not lie in $H^{*}(C) \otimes 1$. So $\nu_{q}$ does not virtually come from $C$, while $E\left(\nu_{q}\right)$ is diffeomorphic to $T \times E\left(\xi_{C}\right)$, and this means that $(C, T, k)$ is not splitting rigid.

If $p_{i}(T C) \in \operatorname{Char}(C, k)$ for all $i>0$, then $D(p(T C))=0$. In other words, $f^{*} p(T C)=p(T C)$, so by Lemma A.1, there is a diffeomorphism $q$ of $C \times T$ such that $q^{*}\left(e\left(\xi_{C}\right) \otimes 1\right)$ does not lie in $H^{*}(C) \otimes 1$. Then the pullback bundle $f^{\#}\left(\xi_{C} \times T\right)$ does not virtually come from $C$, while its total space is diffeomorphic to $T \times E\left(\xi_{C}\right)$; thus $(C, T, k)$ is not splitting rigid.

Remark 9.5. Note that if $k \geq \operatorname{dim}(C)$, then $p_{i}(T C) \in \operatorname{Char}(C, k)$ for all $i>0$.

Remark 9.6. The proof of Proposition 9.4 implies the weak converse of Proposition 3.4 if $(C, T, k)$ is splitting rigid and either $2 k \geq \operatorname{dim}(C \times T)+3$ or $p_{i}(T C) \in$ $C h a r(C, k)$, then any homotopy equivalence of $C \times T$ maps $\operatorname{Char}(C, k) \otimes 1$ to itself.

The following example is due to T. Yamaguchi YYam, Example 3] and it was constructed in response to a question in the previous version of this paper.

Example 9.7. Consider a minimal Sullivan algebra $M$ with the generators $\{x, y, z, a, b, c\}$, whose degrees are respectively $2,3,3,4,5,7$, and the differential given by $d(x)=d(y)=0, d(z)=x^{2}, d(a)=x y, d(b)=x a+y z, d(c)=$ 
$a^{2}+2 y b$. A direct computation shows that the rank of $H^{i}(M)$ is equal to 1 for $i=0,2,3,11,12,14$, is equal to 2 for $i=7$, and is equal to 0 for other $i$ 's. Also the generators of $H^{*}(C)$ as an algebra are

$$
x, y, e=y a, f=x b-z a, g=x^{2} c-x a b+y z b, h=3 x y c+a^{3} .
$$

The products are all trivial, except $x h, y g$, and ef in $H^{14}(M)$. Thus $H^{*}(M)$ satisfies the Poincare duality, and therefore by rational surgery [Sul77, Theorem 13.2], there exists a closed simply-connected elliptic 14-dimensional manifold $C$ with minimal model $M$.

Then $\operatorname{Der}\left(H^{*}(C)\right)$ is not zero since there is, for example, a nonzero derivation $(g, y)$ of degree -8 . Here $(p, q)$ stands for the derivation which send $p$ to $q$ and other generators to zero. One can check that $(g, y)$ is indeed a derivation. On the other hand, another direct computation (see YYam, Example 3]) shows that all derivations induced from $M$ vanish on $H^{*}(C)$. Therefore, the manifold $X=C \times C \times C \times C$ has the same property, but there exists a derivation $D$ of $H^{*}(X)$ of degree -8 which is nonzero on $H^{44}(X)$. Thus, $(X, T, k)$ is splitting rigid for any $T, k$, but this fact cannot be seen by looking only at $H^{*}(X)$.

The following lemma, combined with Proposition 9.1, shows that the property of being "splitting rigid for all $k$ " depends only on $\operatorname{dim}(T)$ and the rational homotopy type of $C$.

Lemma 9.8. Let $(C, T, k)$ be splitting rigid for all $k$. Then if $D$ is a negative derivation of $H^{*}(C)$ of degree $\geq-\operatorname{dim}(T)$ induced by a derivation of $M_{C}$, then $D$ vanishes on $H^{\text {even }}(C)$.

Sketch of the proof. As in the proof of Proposition 9.4 use $D$ to construct a selfhomotopy equivalence $f$ of $C \times T$.

If $D$ is nonzero on $H^{4 i}(C)$ for some $i$, then $D$ is nonzero on the $i$ th Pontrjagin class of some bundle $\xi_{C}$ over $C$. Then for some large $k$, the map $f: C \times T \rightarrow$ $E\left(\xi_{C}\right) \times T$ is homotopic to a smooth embedding. By the same argument as in the proof of Proposition 9.4 the normal bundle to this embedding does not virtually come from $C$ and, therefore, $(C, T, k)$ is not splitting rigid.

If $D$ vanishes on $\bigoplus_{i} H^{4 i}(C)$, then $D(p(T C))=0$ so $f$ preserves $p(T C)$. Hence, by Lemma A.1, replacing $f$ with some power of $f$, we can assume that $f$ is homotopic to a diffeomorphism. If $D$ is nonzero on $H^{2 i}(C)$ for some $i$, then $D$ is nonzero on the Euler class of some bundle $\xi_{C}$ over $C$. Looking at the bundle $f^{\#}\left(\xi_{C} \times T\right)$ shows that $(C, T, 2 i)$ is not splitting rigid.

\section{Proof of Theorem 1.4}

Let $G=S U(6)$ and $H=S U(3) \times S U(3)$. According to [T097, Chapter 5, Example 4.14] (cf. [GHV76, Section 11.14] and [FHT01, Proposition 5.16]), the minimal model of $G / H$ is given by $(M, d)=\left(\Lambda\left(y_{4}, y_{6}, x_{7}, x_{9}, x_{11}\right), d\right)$ with the degrees given by the subscripts, and $d\left(y_{4}\right)=0=d\left(y_{6}\right), d\left(x_{7}\right)=y_{4}^{2}, d\left(x_{9}\right)=2 y_{4} y_{6}$, $d\left(x_{11}\right)=y_{6}^{2}$.

Now it is straightforward to compute the cohomology algebra of $G / H$. In particular, $G / H$ has nonzero Betti numbers only in dimensions $0,4,6,13,15,19$ and the cohomology groups in dimensions 4,6 are generated by the classes $\left[y_{4}\right],\left[y_{6}\right]$.

Let $\xi$ be the rank 3 complex vector bundle over $G / H$ classified by $p: G / H \rightarrow$ $B S U(3)$ which is the composition of the classifying map $G / H \rightarrow B H=B S U(3) \times$ 
$B S U(3)$ for the bundle $G \rightarrow G / H$ with the projection $B S U(3) \times B S U(3) \rightarrow$ $B S U(3)$ on the first factor. Since $G /(S U(3) \times 1)$ is 6 -connected, from the Serre spectral sequence of the bundle $G / S U(3) \rightarrow G / H \rightarrow B S U(3)$ we see that the map $p^{*}: H^{i}(B S U(3)) \rightarrow H^{i}(G / H)$ is an isomorphism for $i \leq 6$. Hence, $c_{3}(\xi)$ is the generator of $H^{6}(G / H)$, and by rescaling $y_{6}$, we can assume that $c_{3}(\xi)=\left[y_{6}\right]$. Note that $c_{3}(\xi)$ is equal to the Euler class $e\left(\xi_{\mathbb{R}}\right)$ of $\xi_{\mathbb{R}}$, the realification of $\xi$.

Alternatively, $\xi$ can be described as the associated bundle to the principal bundle $G \rightarrow G / H$ via the representation $\rho$ of $S U(3) \times S U(3)$ given by the projection onto the first factor followed by the standard action of $S U(3)$ on $\mathbb{C}^{6}$. Thus, $E(\xi)$ admits a complete metric with sec $\geq 0$ such that the zero section is a soul.

To finish the proof, it remains to find a torus $T$ and a self-diffeomorphism $f$ of $C \times T$ such that $f^{*}\left(c_{3}(\xi) \otimes 1\right) \notin H^{*}(G / H) \otimes 1$, because then the bundle $f^{\#} \xi_{\mathbb{R}}$ does not virtually come from $C$, and $E\left(f^{\#} \xi_{\mathbb{R}}\right)$ carries a complete metric with sec $\geq 0$ and zero section being a soul.

Because $M$ is free, the linear map $\tilde{D}: M \rightarrow M$ defined by $\tilde{D}\left(y_{4}\right)=\tilde{D}\left(x_{7}\right)=$ $0, \tilde{D}\left(y_{6}\right)=y_{4}, \tilde{D}\left(x_{9}\right)=x_{7}, \tilde{D}\left(x_{11}\right)=2 x_{9}$ is a derivation of $M$ of degree -2 (see [FHT01 page 141]). By computing on the generators, it is straightforward to see that $\tilde{D}$ commutes with $d$, and hence induces a derivation $D$ of $H^{*}(G / H)$ such that $D\left(\left[y_{6}\right]\right)=\left[y_{4}\right]$.

By Lemma 9.3, there is a positive integer $m$ and a self-homotopy equivalence $h$ of $C \times T$ where $\operatorname{dim}(T)=2$ such that $\pi_{T} \circ h=\pi_{T}$ and $h^{*}(a \otimes 1)=a \otimes 1+$ $\left(1 \otimes t_{3}\right)(m D(a) \otimes 1)$ for any $a \in H^{*}(G / H)$.

Note that $h^{*}$ preserves the total Pontrjagin class of the tangent bundle to $G / H \times$ $T$. Indeed, since $T$ is parallelizable and $H^{4 i}(G / H)$ are only nonzero if $i=0,1$, it suffices to show that $h^{*}$ preserves $p_{1}(G / H) \otimes 1$, or equivalently, that $m D$ vanishes on $p_{1}(G / H)$. In fact, more is true, namely, $D$ vanishes on $H^{4}(G / H)$ since $D\left(\left[y_{4}\right]\right)=$ $\left[\tilde{D}\left(y_{4}\right)\right]=0$. By Lemma A.1 below, some power

$$
f=h^{k}=\underbrace{h \circ \cdots \circ h}_{k}
$$

of $h$ is homotopic to a diffeomorphism. Since $\pi_{T} \circ h=\pi_{T}$, we have $\pi_{T} \circ f=\pi_{T}$ so that $f^{*}(1 \otimes t)=1 \otimes t$ for any $t \in H^{*}(T)$. Combining with $\left(t_{3}\right)^{2}=0$, we get for $a \in H^{*}(C)$

$$
f^{*}(a \otimes 1)=a \otimes 1+\left(1 \otimes t_{3}\right)(k m D(a) \otimes 1) .
$$

Since $D\left(c_{3}(\xi)\right)=D\left(\left[y_{6}\right]\right)=\left[y_{4}\right] \neq 0$, we get $f^{*}\left(c_{3}(\xi) \otimes 1\right) \notin H^{*}(G / H) \otimes 1$, and the proof of Theorem 1.4 is complete.

\section{Proving SPLitTing Rigidity Without Rational homotopy}

This section contains an example of two triples $(C, T, 6),(M, T, 6)$ such that $C$ and $M$ are homotopy equivalent and $(C, T, 6)$ is splitting rigid, while $(M, T, 6)$ is not.

Let $M=S^{3} \times S^{3} \times S^{10} \times S^{11}$. Note that $(M, T, 6)$ is not splitting rigid if $\operatorname{dim}(T) \geq 3$. Indeed, let $\xi_{M}$ be the pullback of $T S^{6}$ via the map $M \rightarrow S^{6}$, which is the composition of the projection $M \rightarrow S^{3} \times S^{3}$ followed by a degree one map $S^{3} \times S^{3} \rightarrow S^{6}$. Then $\xi_{M}$ has nonzero Euler class. If $\operatorname{dim}(T) \geq 3$, then one can easily construct a self-diffeomorphism $f$ of $M \times T$ such that $f^{*} e\left(\xi_{M} \times T\right)$ does not lie in $H^{6}(M) \otimes 1$, in particular, the bundle $f^{\#}\left(\xi_{M} \times T\right)$ does not virtually come from $M$. 
Proposition 11.1. There exists a smooth manifold $C$ homotopy equivalent to $M$ such that $(C, T, 6)$ is splitting rigid for any $T$.

Proof. By a surgery argument as in BK01a, A.1], there is a closed smooth manifold $C$ which is homotopy equivalent to $M$ and has $p(T C)=1+p_{4}(T C)$ with $p_{4}(T M) \neq$ 0 .

To check that $(C, T, 6)$ is splitting rigid, we need to start with an arbitrary rank 6 vector bundle $\xi$ over $B=C \times T$ that satisfies $(*)$ and prove that $\xi$ virtually comes from $C$, or equivalently, that the Euler and Pontrjagin classes of $\xi$ lie in $\operatorname{Char}(C, 6) \otimes 1$.

We shall borrow notation and arguments from the proof of Proposition 3.4. As in Proposition 3.4, we can assume that $E(\xi)$ is the total space of a vector bundle $\eta$ which is the product of $T$ and a vector bundle $\eta_{C^{\prime}}$ over a closed smooth simplyconnected manifold $C^{\prime}$. Let $S=C^{\prime} \times T$ and $g: B \rightarrow S$ by the homotopy equivalence as in Proposition 3.4

Note that $g^{*} p(T S)=p(T B)$. Indeed, as in Proposition 3.4, $g^{*} p\left(\left.T N\right|_{S}\right)=$ $p\left(\left.T N\right|_{B}\right)$. So $g^{*} p(\eta) g^{*} p(T S)=p(\xi) p(T B)$. The only Pontrjagin classes of a rank 6 vector bundle that have a chance of being nonzero are $p_{1}, p_{2}, p_{3}$. Since $C^{\prime}$ has zero cohomology in dimensions $4,8,12$, we get $p(\eta)=1$. By the same argument, $p(T S)=1+p_{4}(T S)+p_{6}(T S)$. We have

$$
g^{*}\left(1+p_{4}(T S)+p_{6}(T S)\right)=\left(1+p_{1}(\xi)+p_{2}(\xi)+p_{3}(\xi)\right)\left(1+p_{4}(T B)\right),
$$

hence $p_{6}(T S)=0=p_{i}(\xi)$ for all $i$, and $g^{*} p(T S)=p(T B)$. Then one easily sees that $g_{C C^{\prime}}$ maps $p\left(T C^{\prime}\right)$ to $p(T C)$. Hence, $g_{C C^{\prime}} \times g_{T T}$ maps $p(T S)$ to $p(T B)$, and therefore, $h=\left(g_{C C^{\prime}}^{-1} \times g_{T T}^{-1}\right) \circ g$ preserves $p(T B)$.

As in Proposition 3.4. it suffices to show that $h$ preserves $\operatorname{Char}(C, 6) \otimes 1=$ $H^{6}(C) \otimes 1$. We think of $H^{*}(C)$ as an exterior algebra on generators $x, y, q, s$ corresponding to spheres $S^{3}, S^{3}, S^{10}, S^{11}$ so we need to show that $h^{*}(x y)=x y$. By rescaling, we can assume that $p(T B)=x y q \otimes 1$.

By dimension reasons $\frac{\partial h^{*}}{\partial t_{i}}(x y)=0$ unless $\left|t_{i}\right|$ is 3 or 6 . Similarly, $\frac{\partial h^{*}}{\partial t_{i}}(q)=0$ unless $\left|t_{i}\right|$ is 4,7 or 10 . Now collecting terms next to $1 \otimes t_{i}$ 's in the identity $x y q \otimes 1=h^{*}(x y q \otimes 1)$, we conclude that $q \frac{\partial h^{*}}{\partial t_{i}}(x y)=0$, and hence $\frac{\partial h^{*}}{\partial t_{i}}(x y)=0$ for all $i>0$. Thus, $h^{*}(x y)=x y$ as promised.

Remark 11.2. The above example shows that Proposition9.4 fails without assuming either $2 k \geq \operatorname{dim}(C \times T)+3$ or $p_{i}(T C) \in \operatorname{Char}(C, k)$. Indeed, $\left(H^{*}(C), 0\right)$ is a minimal model of $C$ and there is a degree -3 derivation of $H^{*}(C)$ given by $D(x)=1$, $D(y)=D(q)=D(s)=0$ which does not vanish on $\operatorname{Char}(C, 6)=H^{6}(C)$. Namely, $D(x y)=y$. Yet $(C, T, 6)$ is splitting rigid.

It is instructive to see where the proof of Proposition 9.4 fails. Using $D$, we produce a self-homotopy equivalence $f$ of $C \times T$ and an $\mathbb{R}^{6}$-bundle $\xi_{C}$ with $x y=$ $e\left(\xi_{C}\right) \otimes 1$. However, the homotopy equivalence $f: C \times T \rightarrow E\left(\xi_{C}\right) \times T$ is not homotopic to a smooth embedding.

Remark 11.3. As always with splitting rigid triples, the total spaces of "most" $\mathbb{R}^{6}$ bundles over $C \times T$ do not admit complete metrics with sec $\geq 0$. We do not know whether $C$ in Proposition 11.1 admits a metric with sec $\geq 0$. Yet, no currently known method rules out the existence of sec $\geq 0$ on $C$, because $C$ is homotopy equivalent to a closed nonnegatively curved manifold, and $C$ admits a metric of 
positive scalar curvature, for $C$ is spin and $\operatorname{dim}(C)=27 \equiv 3(\bmod 8)$, so Sto92] applies.

\section{Nonnegatively CURVED VeCtor BUndLes WITH SOULS EQUAL TO THE ZERO SECTIONS}

The purpose of this section is to obtain restrictions on normal bundles to souls in nonnegatively curved manifolds. In other words, we look for conditions on a vector bundle $\xi$ ensuring that $E(\xi)$ admits no complete nonnegatively curved metric such that the zero section is a soul. The assumption that a given submanifold is a soul imposes a nontrivial restriction on the metric, so it is no surprise that we get stronger results on obstructions.

Our exposition is parallel to the one in Section 3. We say that a vector bundle $\xi$ over $C \times T$ satisfies condition $(* *)$ if

there is a finite cover $\pi: C \times T \rightarrow C \times T$, a closed manifold $C^{\prime}$, and a diffeomorphism $f: C^{\prime} \times T \rightarrow C \times T$ such that the bundle $f^{\#} \pi^{\#}(\xi)$ virtually comes from $C^{\prime}$.

Example 12.1. According to Theorem [3.1, if $S$ is a soul in a complete nonnegatively curved manifold, then the normal bundle to $S$ satisfies (**).

Caution. Clearly, if $\xi$ satisfies $(* *)$, it also satisfies condition $(*)$ from Section 3 The converse is generally false, as the following example shows.

Example 12.2. Let $M=S^{3} \times S^{5} \times S^{7}$. By a surgery argument as in [BK01a, A.1], there is a closed 15-dimensional manifold $C$ which is homotopy equivalent to $M$ and such that $p_{2}(T C), p_{3}(T C)$ are nonzero. Clearly, there is a derivation of $H^{*}(C)$ of degree -3 which is nonzero on $H^{8}(C)$. Since $\left(H^{*}(C), 0\right)$ is the minimal model for $C$, Proposition 9.4 implies that $(C, T, 15)$ is not splitting rigid if $\operatorname{dim}(T) \geq 3$. Thus, there is a rank 15 bundle $\xi$ over $C \times T$ which satisfies $(*)$ but which does not virtually come from $C$. It remains to show that $\xi$ does not satisfy $(* *)$. If it does, then after passing to a finite cover, we can assume that, for some diffeomorphism $f: C^{\prime} \times T \rightarrow C \times T, f^{\#} \xi$ virtually comes from $C^{\prime}$. Since $f$ is a diffeomorphism and $T$ is parallelizable, $f^{*}\left(p_{i}(T C) \otimes 1\right)=p_{i}\left(T C^{\prime}\right) \otimes 1$ for all $i$. Since $p_{i}(T C)$ generates $H^{4 i}(C)$ and $f^{*}$ is an isomorphism, this means that $f^{*}\left(H^{4 i}(C) \otimes 1\right)=H^{4 i}\left(C^{\prime}\right) \otimes 1$ for all $i$. Since $f^{*} p_{i}(\xi)=p_{i}\left(f^{\#}(\xi)\right) \in H^{4 i}\left(C^{\prime}\right) \otimes 1$ for all $i$, this implies that $p_{i}(\xi) \in$ $H^{4 i}(C) \otimes 1$ for all $i$ and hence $\xi$ virtually comes from $C^{\prime}$. This is a contradiction, so $\xi$ cannot satisfy $(* *)$.

Recall that, given a compact Lie group $G$, a rank $n$ vector bundle $\xi$ has structure group $G$ if $\xi$ is associated with a principal $G$-bundle via some representation $G \rightarrow$ $O(n)$. We now have the following splitting criterion similar to Proposition 3.4:

Proposition 12.3. Let $\xi$ be a vector bundle over $C \times T$ which has structure group $O(k)$ and satisfies (**). If any self homotopy equivalence of $C \times T$ maps $C h a r(C, k) \otimes 1$ to itself, then $\xi$ virtually comes from $C$.

Proof. Passing to a finite cover, we can assume that $\xi$ is orientable and that $\eta=$ $f^{\#} \xi$ virtually comes from $C^{\prime}$, where $f: C^{\prime} \times T \rightarrow C \times T$ is a diffeomorphism. As before, to show that $\xi$ virtually comes from $C$, it is enough to check that its rational characteristic classes lie in $H^{*}(C) \otimes 1$. Proceeding exactly as in the proof of Proposition 3.4. we conclude that $\left(f^{*}\right)^{-1}$ maps $\operatorname{Char}\left(C^{\prime}, k\right) \otimes 1$ to $\operatorname{Char}(C, k) \otimes 1$. 
Next note that all $e(\eta), p_{i}(\eta)$ lie in the subalgebra generated by $C h a r\left(C^{\prime}, k\right) \otimes 1$. Indeed, $\eta$ has the structure group $S O(k)$, so $\eta$ is a pullback of a bundle over $B S O(k)$. Since the cohomology of $B S O(k)$ is generated by $\operatorname{Char}(B S O(k), k)$, by naturality of the characteristic classes, we see that $e(\eta), p_{i}(\eta) \in\left\langle\operatorname{Char}\left(C^{\prime}, k\right)\right\rangle$ for any $i$.

Since $\left(f^{*}\right)^{-1}\left(\left\langle C h a r\left(C^{\prime}, k\right) \otimes 1\right\rangle\right) \subset\langle C h a r(C, k) \otimes 1\rangle$, we conclude that all the characteristic classes of $\xi$ lie in $\langle\operatorname{Char}(C, k) \otimes 1\rangle \subset H^{*}(C) \otimes 1$, hence $\xi$ virtually comes from $C$.

Now all the splitting rigidity results that relied on Proposition 3.4 can be adapted to this new setting. In particular, we obtain

Theorem 12.4. Let $\xi$ be a vector bundle over $C \times T$ with structure group $O(2)$. If $E(\xi)$ admits a complete metric with $\mathrm{sec} \geq 0$ such that the zero section is a soul, then $\xi$ virtually comes from $C$.

Theorem 12.5. Let $C=G / / H$ be a simply connected biquotient of compact Lie groups such that $H$ is semisimple. Let $\xi$ be a vector bundle over $C \times T$ whose structure group can be reduced to a subgroup of $O(4)$. If $E(\xi)$ admits a complete metric with $\mathrm{sec} \geq 0$ such that the zero section is a soul, then $\xi$ virtually comes from C.

\section{OPEN PROBLEMS}

13.1. Induced derivations. As we proved in Section 9 for sufficiently large $k$, splitting rigidity of $(C, T, k)$ is equivalent to vanishing on $C h a r(C, k)$ of all negative derivations of degree $\geq-\operatorname{dim}(T)$ induced from the minimal model $M_{C}$. Yet all our geometric applications are proved by checking the stronger condition that all negative derivations vanish on $\operatorname{Char}(C, k)$. This is mostly due to the fact that we do not know how to effectively check which derivations of $H^{*}(C)$ are induced from the minimal model.

Let us restate the problem in purely rational homotopy theoretic terms. It is well known that the space $\operatorname{Der}\left(M_{C}\right)$ is a differential graded Lie algebra (DGLA) with the differential given by $D_{C}=\left[-, d_{C}\right]$. It is trivial to check that closed derivations preserve $\operatorname{ker}\left(d_{C}\right)$ and exact ones send $\operatorname{ker}\left(d_{C}\right)$ to $\operatorname{Im}\left(d_{C}\right)$ (see Gri94 for details). Therefore, we have a natural graded Lie algebra homomorphism $m: H^{*}\left(\operatorname{Der}\left(M_{C}\right)\right) \rightarrow \operatorname{Der}\left(H^{*}(C)\right)$. We seek to understand the image of the map $m$. Example 9.7 produces an elliptic smooth manifold $C$ such that $\operatorname{Im}(m)=0$ but $\operatorname{Der}\left(H^{*}(C)\right) \neq 0$.

To relate to our geometric applications we would like to find such examples when $C$ is nonnegatively curved. It is easy to see that $m$ is onto if $C$ is formal, but that is all we can generally say at the moment. (Recall that a space $X$ is called formal if $X$ and $\left(H^{*}(X), d=0\right)$ have isomorphic minimal models.)

Let us also mention that according to Sullivan [Sul77], the DGLA $\left(\operatorname{Der}\left(M_{C}\right), D_{C}\right)$ is a (Quillen) Lie algebra model for $\operatorname{Baut}_{1}(C)$ (the classifying space for the identity component of the monoid of self-homotopy equivalences of $C$ ), and therefore, understanding the map $m$ can be helpful for computing the rational homotopy groups of $\operatorname{Baut}_{1}(C)$.

13.2. Halperin's conjecture for biquotients. As we mentioned in the introduction, the conjecture of Halperin that any elliptic space of positive Euler characteristic belongs to $\mathcal{H}$ has been verified for all homogeneous spaces of compact 
Lie groups ST87. However, it remains open for the natural bigger class of elliptic spaces formed by biquotients. According to [Sin93], a biquotient $G / / H$ has a positive Euler characteristic iff $\operatorname{rank}(G)=\operatorname{rank}(H)$. Therefore, we pose the following

Problem 13.1. Prove that any biquotient $G / / H$ belongs to $\mathcal{H}$ if $\operatorname{rank}(G)=$ $\operatorname{rank}(H)$.

This is unknown even for the simplest examples such as $S p(1) \backslash S p(n) / S U(n)$.

13.3. Nonnegatively curved vector bundles over rational $\mathbf{H}$-spaces. Most explicit examples of nonnegatively curved bundles are given by homogeneous vector bundles, i.e., by vector bundles associated to principal $H$-bundles $H \rightarrow G \rightarrow G / H$ via some representations $H \rightarrow O(k)$. For any given $k$, there are only finitely many rank $k$ homogeneous vector bundles (because the number of nonequivalent irreducible representations $H \rightarrow O(k)$ is finite). However, homogeneous vector bundles can fill a substantial part of $[G / H, B O]$, the set of stable equivalence classes of bundles over $G / H$.

If $H=1$, or more generally if $G / H$ is rationally homotopy equivalent to the product of odd-dimensional spheres, then $H^{*}(B H) \rightarrow H^{*}(G / H)$ has trivial image [GHV76, page 466], and hence, any homogeneous vector bundle over $G / H$ has zero Euler and Pontrjagin classes. Motivated by the above discussion, we pose the following:

Problem 13.2. Does there exist a nonnegatively curved vector bundle $\xi$ over a closed manifold $C$ such that $C$ is rationally homotopy equivalent to the product of odd-dimensional spheres, $\sec (C) \geq 0$, and $e(\xi) \neq 0$ or $p_{i}(\xi) \neq 0$ for some $i>0$ ?

13.4. Nonnegatively curved nonsplitting rigid examples. The example of a nonnegatively curved vector bundle $\xi$ that satisfies condition $(*)$ but does not virtually come from $C$ provided by Theorem 1.4 is essentially the only example of this kind known to us. This is not very satisfactory, for instance, because this example is unstable; that is, $\xi \oplus \epsilon^{1}$ does virtually come from $C$. More importantly, we want to understand how "generic" such examples are.

To construct a stable example, it suffices to find a vector bundle $\xi$ over $C$ with $\sec (E(\xi)) \geq 0$ and soul equal to the zero section, and a negative derivation $D$ of $H^{*}(C)$ induced by a derivation of the minimal model such that $D(p(\xi)) \neq 0$ and $D(p(T C))=0$. While we think that many such examples exist, finding an explicit one, say among homogeneous vector bundles, seems to be an unpleasant task because

(1) Pontrjagin classes of homogeneous vector bundles are often difficult to compute, and

(2) there is no easy algorithm for computing the space of negative derivations of $H^{*}(G / H)$ or of the minimal model of $G / H$.

One of the few cases when $\operatorname{Der}_{-}\left(H^{*}(G / H)\right)$ is easily computable is when $G / H$ is formal. According to [Oni94 Theorem 12.2], any formal compact homogeneous space $G / H$ is rationally homotopy equivalent to the product of odd-dimensional spheres and an elliptic space $X$ of positive Euler characteristic. Again by Oni94, Theorem 12.2], the image of the homomorphism $H^{*}(B H) \rightarrow H^{*}(G / H)$ is equal to the $H^{*}(X)$-factor. If $X \in \mathcal{H}$ (i.e., if Halperin's conjecture holds for $X$ ), then by Lemma [6.1, one concludes that if $\xi$ is a homogeneous vector bundle and $D$ is a negative derivation of $H^{*}(G / H)$, then $D$ vanishes on $e(\xi), p_{i}(\xi)$ for $i>0$. 
Thus, if Halperin's conjecture is true, then homogeneous vector bundles over formal homogeneous spaces cannot be used to prove an analog of Theorem 1.4

Finally, note that a positive solution to Problem 13.2 (for $C$ with $p(T C)=1$ which includes the case when $C$ is a compact Lie group or the product of odddimensional spheres) yields an analog of Theorem 1.4 because for any nontrivial element $a$ of $H^{*}(C)$, there exists a negative derivation $D$ of $H^{*}(C)$ with $D(a) \neq 0$, and $D(p(T C))$ vanishes by assumption.

\section{Appendix A. Surgery-theoretic LEMma}

We are grateful to Ian Hambleton for sketching the proof of the following lemma.

Lemma A.1. Let $C$ be a closed smooth simply-connected manifold, and let $T$ be a torus such that $\operatorname{dim}(C)+\operatorname{dim}(T) \geq 5$. If $h$ is a self-homotopy equivalence of $C \times T$ that preserves the rational total Pontrjagin class of $C \times T$, then $h^{m}$ is homotopic to a diffeomorphism for some $m>0$.

Proof. Let $k=\operatorname{dim}(T), n=\operatorname{dim}(C), I=[0,1], B=C \times T, \pi=\pi_{1}(B)$ so that $\pi \cong \mathbb{Z}^{k}$. Look at the following commutative diagram whose rows are the smooth and the topological surgery exact sequences:

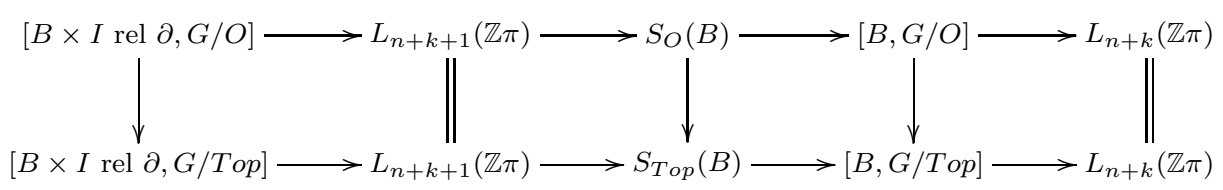

First, note that $[B \times I$ rel $\partial, G / T o p] \rightarrow L_{n+k+1}(\mathbb{Z} \pi)$ is onto. Indeed, by the Poincare duality with $L$-theory coefficients $[B \times I$ rel $\partial, G / T o p]=H^{0}(B \times I ; \mathbf{L}) \cong$ $H_{n+k+1}(B \times I ; \mathbf{L})$, so it suffices to show that the homology assembly map (see, e.g., Dav94, page 216])

$$
A_{n+k+1}: H_{n+k+1}(B \times I ; \mathbf{L}) \rightarrow L_{n+k+1}(\mathbb{Z} \pi)
$$

is onto. By naturality of the assembly and since $T$ is the classifying space for $\pi_{1}(B \times I), A_{n+k+1}$ factors as the composition of the map $H_{n+k+1}(B \times I ; \mathbf{L}) \rightarrow$ $H_{n+k+1}(T ; \mathbf{L})$ induced by the projection $B \times I \rightarrow T$, and the universal assembly $H_{n+k+1}(T ; \mathbf{L}) \rightarrow L_{n+k+1}(\mathbb{Z} \pi)$. The former map is onto, since it has a section induced by a section of $B \times I \rightarrow T$, while the latter map is an isomorphism since $\pi \cong \mathbb{Z}^{k}$ [Wal99, Chapter 15B]. Thus, $A_{n+k+1}$ is onto.

It is known that the map $[B \times I$ rel $\partial, G / O] \rightarrow[B \times I$ rel $\partial, G / T o p]$ has a finite cokernel (see, e.g., Dav94, page 213]), and hence so does the map $[B \times I$ rel $\partial, G / O] \rightarrow L_{n+k+1}(\mathbb{Z} \pi)$.

By exactness of the smooth surgery exact sequence, the $L_{n+k+1}(\mathbb{Z} \pi)$-action on $S_{O}(B)$ has finite orbits.

Since $h$ preserves the total Pontrjagin class, $h^{m}$ is tangential for some $m>0$, so replacing $h$ by $h^{m}$, we can assume that $h$ is tangential. Hence for any integer $l>0$, $h^{l}$ is tangential so that the normal invariant of $\left[B, h^{l}\right] \in S_{O}(B)$ lies in the image of the map $[B, S G] \rightarrow[B, G / T o p]$ induced by the fibration $S T o p \rightarrow S G \rightarrow G / T o p$. Since $S G$ is rationally contractible, $[B, S G]$ is a finite set, so there exists an infinite sequence of positive integers $l_{k}$ such that the elements $\left[B, h^{l_{k}}\right] \in S_{O}(B)$ have the same normal invariant. By exactness, $\left[B, h^{l_{k}}\right]$ lie in the same $L_{n+k+1}(\mathbb{Z} \pi)$-orbit which is a finite set by above. In particular, for some $p>q>0$ we have $\left[B, h^{p}\right]=$ 
$\left[B, h^{q}\right]$. Thus, for some self-diffeomorphism $f$ of $B$, we get that $f h^{q}$ and $h^{p}$ are homotopic, or $f$ is homotopic to $h^{p-q}$, as wanted.

\section{ApPendix B. VeCtor BUNDLES WITH PRESCRIBED CHARACTERISTIC ClAsses}

The following lemma is probably well known; yet there seems to be no reference available, so we include a complete proof.

Lemma B.1. Let $X$ be a finite $C W$-complex, and let $n$ be a positive integer.

(i) If $k=2 n+1$, then for any $n$-tuple $\left(p_{1}, \ldots, p_{n}\right)$ of cohomology classes with $p_{i} \in H^{4 i}(X)$ for $i=1, \ldots, n$, there is an integer $m>0$ and an orientable rank $k$ vector bundle $\xi$ over $X$ such that $p_{i}(\xi)=m p_{i}$ for $i=1, \ldots, n$.

(ii) If $k=2 n$, then for any $n$-tuple $\left(p_{1}, \ldots, p_{n-1}, e\right)$ of cohomology classes with $p_{i} \in H^{4 i}(X)$ for $i=1, \ldots, n-1$, and $e \in H^{2 n}(X)$, there is an integer $m>0$ and an orientable rank $k$ vector bundle $\xi$ over $X$ such that $e(\xi)=m e, p_{i}(\xi)=m p_{i}$ for $i=1, \ldots, n-1$.

Proof. We only give a proof for $k=2 n+1$; the even case is similar. Let $\gamma^{k}$ be the universal $k$-bundle over $B S O(k)$. We think of $p_{i}\left(\gamma^{k}\right) \in H^{4 i}(B S O(k)) \cong$ $[B S O(k), K(\mathbb{Q}, 4 i)]$ as a map $B S O(k) \rightarrow K(\mathbb{Q}, 4 i)$. It is well known that the map

$$
c=\left(p_{1}\left(\gamma^{k}\right), \ldots, p_{n}\left(\gamma^{k}\right)\right): B S O(k) \rightarrow K=K(\mathbb{Q}, 4) \times \cdots \times K(\mathbb{Q}, 4 n)
$$

is a rational homotopy equivalence, and thus the homotopy groups of its homotopy fiber $F$ are torsion.

Similarly, consider the map $f=\left(p_{1}, \ldots, p_{n}\right): X \rightarrow K$ and try to lift it to $B S O(k)$. In other words, try to find $\tilde{f}: X \rightarrow B S O(n)$ which would make the following diagram commute up to homotopy:

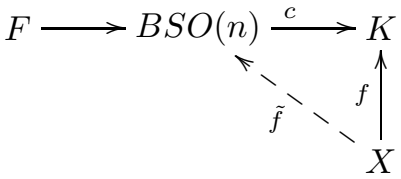

The obstructions to lifting $f$ lie in torsion groups $H^{*+1}\left(X,\left\{\pi_{*}(F)\right\}\right)$ and are generally nonzero.

Each factor $K(\mathbb{Q}, i)$ of $K$ is an $H$-space, so let $\times_{i}^{m}: K(\mathbb{Q}, i) \rightarrow K(\mathbb{Q}, i)$ be the $m$ th power map. It is easy to check that the endomorphism of $H^{i}(K(\mathbb{Q}, i), \mathbb{Z})$ induced by $\times^{m}$ is the multiplication by $m$. The $\mathbb{Q}$-algebra $H^{*}(K(\mathbb{Q}, i), \mathbb{Z})$ is generated in dimension $i$, so we get an obvious generating set for $H^{*}(K, \mathbb{Z})$ such that the $m$ th power map $\times^{m}=\prod_{i} \times_{i}^{m}$ of $K$ acts on the generators as the multiplication by $m$.

Let $o_{j}$ be the first nontrivial obstruction to lifting $f$. Since $o_{j}$ is torsion and $X$ is a finite $\mathrm{CW}$-complex, the naturality of obstructions implies that $o_{j}\left(\times^{m} \circ f\right)=0$ for some $m$. Repeating this process finitely many times, we find some $m$ such that all the obstructions to lifting $\times^{m} \circ f$ vanish, and thus there is a map $\tilde{f}: X \rightarrow B S O(n)$ such that $c \circ \tilde{f}$ is homotopic to $\times^{m} \circ f$.

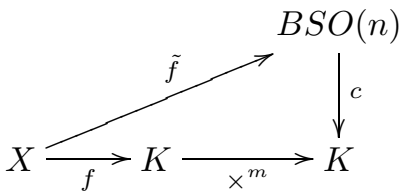

Now the bundle $\xi=\tilde{f}^{\#}\left(\gamma^{n}\right)$ has the desired properties. 


\section{ACKNOWLEDGMENTS}

The first author is grateful to McMaster University and California Institute of Technology for support and excellent working conditions.

It is our pleasure to thank Gregory Lupton and Samuel Smith for insightful discussions on rational homotopy theory, Stefan Papadima for Lemma 5.1 Ian Hambleton for Lemma A.1. Toshihiro Yamaguchi for Example 9.7 Alexander Givental for incisive comments on deformation theory, and Burkhard Wilking and Wolfgang Ziller for countless discussions and insights related to this work. We are grateful to the referee for helpful advice on the exposition. As always, the authors are solely responsible for possible mistakes.

The present paper grew out of our earlier preprint [BK] written in the summer of 2000. In BK] we proved much weaker results, for example, Theorem 1.3 is stated there as an open question. Most of the results of the present paper were obtained in May and early June of 2001 and reported by the first author during the Oberwolfach geometry meeting on June 12, 2001. On June 29, 2001, we received a preprint by Jianzhong Pan where he independently proves Theorem 1.3 in response to our question in [BK].

\section{REFERENCES}

[Baz96] Ya. V. Bazalkin, On a family of 13-dimensional closed Riemannian manifolds of positive curvature, Sibirsk. Mat. Zh. 37 (1996), no. 6, 1219-1237, ii. MR 98c:53045

[BK] I. Belegradek and V. Kapovitch, Obstructions to nonnegative curvature and rational homotopy theory, preprint, 2000, available electronically at the xxx-achive: http://arxiv.org/abs/math.DG/0007007.

[BK01a] I. Belegradek and V. Kapovitch, Finiteness theorems for nonnegatively curved vector bundles, Duke Math. J. 108 (2001), no. 1, 109-134. MR 2002c:53050

[BK01b] I. Belegradek and V. Kapovitch, Topological obstructions to nonnegative curvature, Math. Ann. 320 (2001), no. 1, 167-190. MR 2002d:53044

[CG72] J. Cheeger and D. Gromoll, On the structure of complete manifolds of nonnegative curvature, Ann. of Math. 96 (1972), 413-443. MR 46:8121

[Che73] J. Cheeger, Some examples of manifolds of nonnegative curvature, J. Differential Geom. 8 (1973), 623-628. MR 49:6085

[Dav94] J. Davis, Manifold aspects of the Novikov conjecture, Princeton Univ. Press, 1994, Surveys on surgery theory, Vol. 1, 195-224, Ann. of Math. Stud., 145. MR 2002a:57037

[Esc82] J.-H. Eschenburg, New examples of manifolds with strictly positive curvature, Invent. Math. 66 (1982), no. 3, 469-480. MR 83i:53061

[Esc92a] J.-H. Eschenburg, Cohomology of biquotients, Manuscripta Math. 75 (1992), no. 2, 151-166. MR 93e:57070

[Esc92b] J.-H. Eschenburg, Inhomogeneous spaces of positive curvature, Differential Geom. Appl. 2 (1992), no. 2, 123-132. MR 94j:53044

[Fél89] Y. Félix, La dichotomie elliptique-hyperbolique en homotopie rationnelle, Astérisque (1989), no. 176, 1-187. MR 91c:55016

[FHT01] Y. Félix, S. Halperin, and J.-C. Thomas, Rational homotopy theory, Springer-Verlag, 2001. MR 2002d:55014

[GH83] K. Grove and S. Halperin, Contributions of rational homotopy theory to global problems in geometry, Inst. Hautes Études Sci. Publ. Math. 56 (1982) (1983), 171-177. MR 84b:58030

[GHV76] W. Greub, S. Halperin, and R. Vanstone, Connections, curvature, and cohomology, Academic Press, 1976, Volume III: Cohomology of principal bundles and homogeneous spaces, Pure and Applied Mathematics, Vol. 47-III. MR 53:4110

[Gri94] P.-P. Grivel, Algèbres de Lie de dérivations de certaines algèbres pures, J. Pure Appl. Algebra 91 (1994), no. 1-3, 121-135. MR 95a:55024

[GW00] L. Guijarro and G. Walschap, The metric projection onto the soul, Trans. Amer. Math. Soc. 352 (2000), 55-69. MR 2000c:53034 
[GZ00] K. Grove and W. Ziller, Curvature and symmetry of Milnor spheres, Ann. of Math. (2) 152 (2000), no. 1, 331-367. MR 2001i:53047

[Hae61] A. Haefliger, Plongements différentiables de variétés dans variétés, Comment. Math. Helv. 36 (1961), 47-82. MR 26:3069

[Hal77] S. Halperin, Finiteness in the minimal models of Sullivan, Trans. Amer. Math. Soc. 230 (1977), 173-199. MR 57:1493

[Mar90] M. Markl, Towards one conjecture on collapsing of the Serre spectral sequence, Proceedings of the Winter School on Geometry and Physics (Srní, 1989); Rend. Circ. Mat. Palermo (2) Suppl., no. 22, 1990, pp. 151-159. MR 91c:55033

[Mei83] W. Meier, Some topological properties of Kähler manifolds and homogeneous spaces, Math. Z. 183 (1983), no. 4, 473-481. MR 84j:55005

[MZ87] J. McCleary and W. Ziller, On the free loop space of homogeneous spaces, Amer. J. Math. 109 (1987), 765-781. MR 88k:58023

[Oni94] A. L. Onishchik, Topology of transitive transformation groups, Johann Ambrosius Barth Verlag GmbH, 1994. MR 95e:57058

[ÖW94] M. Özaydin and G. Walschap, Vector bundles with no soul, Proc. Amer. Math. Soc. 120 (1994), no. 2, 565-567. MR 94d:53057

[Rig78] A. Rigas, Geodesic spheres as generators of the homotopy groups of O, BO, J. Differential Geom. 13 (1978), no. 4, 527-545 (1979). MR 81e:57043

[Sin93] W. Singhof, On the topology of double coset manifolds, Math. Ann. 297 (1993), no. 1, 133-146. MR 94k:57054

[ST87] H. Shiga and M. Tezuka, Rational fibrations, homogeneous spaces with positive Euler characteristics and Jacobians, Ann. Inst. Fourier (Grenoble) 37 (1987), no. 1, 81-106. MR 89g:55019

[Sto92] S. Stolz, Simply connected manifolds of positive scalar curvature, Ann. of Math. 136 (1992), no. 3, 511-540. MR 93i:57033

[Sul77] D. Sullivan, Infinitesimal computations in topology, Inst. Hautes Études Sci. Publ. Math. (1977), no. 47, 269-331 (1978). MR 58:31119

[TO97] A. Tralle and J. Oprea, Symplectic manifolds with no Kähler structure, Springer-Verlag, 1997. MR 9k:53038

[Wal72] N. R. Wallach, Compact homogeneous riemannian manifolds with strictly positive curvature, Ann. of Math. 96 (1972), 277-295. MR 46:6243

[Wal99] C. T. C. Wall, Surgery on compact manifolds, second ed., American Mathematical Society, 1999, edited and with a foreword by A. A. Ranicki. MR 2002a:57089

[Wil00] B. Wilking, On fundamental groups of manifolds of nonnegative curvature, Differential Geom. Appl. 13 (2000), no. 2, 129-165. MR 2001g:53076

[Wil02] B. Wilking, Manifolds with positive sectional curvature almost everywhere, Invent. Math. 148 (2002), no. 1, 117-141. MR 2003a:53049]

[Yam] T. Yamaguchi, A rational condition on fiber in fibrations, preprint, 2002.

[Yan95] D. Yang, On complete metrics of nonnegative curvature on 2-plane bundles, Pacific J. Math. 171 (1995), no. 2, 569-583. MR 96k:53034

Department of Mathematics, 253-37, California Institute of Technology, Pasadena, CALIFORNiA 91125

E-mail address: ibeleg@its.caltech.edu

Department of Mathematics, University of California Santa Barbara, Santa BarBARA, CALIFORNia 93106

E-mail address: vitali@math.ucsb.edu 